\title{
ON THE HIGHER DERIVATIVES AT THE BOUNDARY IN CONFORMAL MAPPING $\dagger$
}

BY

\author{
STEFAN E. WARSCHAWSKI
}

\section{INTRODUCTION}

Let $R$ be a region bounded by a closed Jordan curve $C$, and $w=f(z)$ a function which maps the unit circle $|z|<1$ conformally on $R$. As we know, $f(z)$ is then continuous over the circle $|z| \leqq 1$. In an earlier paper $\ddagger$ we have investigated the conditions under which $f(z)$ is differentiable at a boundary point $z_{1}\left(w_{1}=f\left(z_{1}\right)\right)$, that is,

$$
\lim _{z \rightarrow z_{1}} \frac{f(z)-f\left(z_{1}\right)}{z-z_{1}}=f^{\prime}\left(z_{1}\right)
$$

exists for unrestricted approach in $|z| \leqq 1, z \neq z_{1}$, and in addition the conditions under which $f^{\prime}(z)$ is continuous at each point of an arc of $|z|=1$. In the present paper we consider the corresponding questions for the higher derivatives and obtain results of similar nature, of which the following are the principal ones. Let $\Theta(s)$ be an angle from the direction of the positive axis of reals to the tangent line, where $s$ denotes arc length: Let

$$
\kappa^{(n)}(s)=d^{n} \Theta(s) / d s^{n}
$$

be called the curvature of order $n$. If further

$$
\lim \frac{\kappa^{(n-1)}(s)-\kappa^{(n-1)}\left(s^{\prime}\right)}{s-s^{\prime}}
$$

exists when $s$ and $s^{\prime}\left(s \neq s^{\prime}\right)$ approach $s_{1}$ simultaneously, we say that $C$ has an $L$-curvature of order $n$ at $s_{1} . \S$

I. If $C$ has an L-curvature of order $(n-1)$ at $w_{1}: s=s_{1}$, and if

$$
\int_{0}^{a}\left|\kappa^{(n-2)}(s+t)+\kappa^{(n-2)}(s-t)-2 \kappa^{(n-2)}(s)\right| \frac{d t}{t^{2}}
$$

converges for $s=s_{1}$, and if further $w_{1}=f\left(z_{1}\right)$, then $f^{(n-1)}(z)$ assumes continuous boundary values in a neighborhood of $z=z_{1}$, and is differentiable at $z_{1}$. (Theorem IV.)

$\dagger$ Presented to the Society, October 27, 1934; received by the editors November 8, 1934.

$\ddagger$ Mathematische Zeitschrift, vol. 35 (1932), pp. 321-456. We refer to this as WR.

$\S$ The idea of the $L$-curvature generalizes that of the $L$-tangent. The idea of the $L$-tangent was introduced by $E$. Lindelöf, the name " $L$-tangent" by A. Ostrowski. Cf. p. 312. 
II. If $C$ has continuous curvature of order $(n-1)$ along an open arc $c$, and (*) approaches zero uniformly with a on every closed subarc of $c$, then $f^{(n)}(z)$ assumes continuous boundary values on the arc $\gamma$ which corresponds to $c$. (Theorem III (b).)

An extension of II in the case $c \equiv C$ shows how the modulus of continuity $\dagger$ of $f^{(n)}(z)$ on $|z|=1$ depends on the given function $\kappa^{(n-1)}(s)$ and on some other simple properties of $C$ (Theorem III (c)). Thus we obtain a result about the equicontinuity of the $n$th derivatives of the mapping functions at the boundary, for a family of curves which satisfy certain common conditions.

As is well known, the mapping function $f(z)$ varies continuously in $|z| \leqq 1$ under a suitable continuous deformation of $C$. R. Courant, $\ddagger$ T. Radó $\S$ and the author\| have given conditions under which this is true. By means of the above-mentioned extension of II we prove an analogous result for the derivatives (Theorem V).

Earlier results on the higher derivatives of the mapping function were obtained by P.Painlevé, T O.D. Kellogg, †† and W. Seidel. $¥$ In all these cases the hypotheses involve an entire arc of the complete curve, whereas in I above we impose conditions merely at one point. The result of Painlevé, which infers the existence and continuity of $f^{(n)}(z)$ at $|z|=1$ from the continuity of $\kappa^{(n+1)}(s)$ on $C$, is a corollary of II. Kellogg's first paper yields the result that if $\kappa^{(n-1)}(s)$ exists and satisfies a Hölder condition, then $f^{(n)}(z)$ satisfies such a condition with the same exponent at the boundary. This result does not imply any of our results, nor is it implied by any of them. However, it can also be obtained by a modification of our method of proving Theorem III.§§ On the other hand the results of his second paper are easily seen to entail a special case of II. \|\| Seidel proves that, if $\kappa^{(n-2)}(s)$ is absolutely continuous on $C$ and $\left|\kappa^{(n-1)}(s)\right|^{p}(p>1)$ is $L$-integrable, then $f^{(n-1)}(z)$ assumes absolutely continuous boundary values on $|z|=1, f^{(n)}(z)$ has radial boundary values, $f^{(n)}\left(e^{i \theta}\right)$, almost everywhere on $|z|=1$, and $\left|f^{(n)}\left(e^{i \theta}\right)\right|^{p}$ is $L$-integrable. This result neither contains any of our theorems nor is it contained in any of them.

$\dagger$ If $\phi(x)$ is defined and continuous on a closed interval $I$ so that for every $\epsilon>0$ there is a $\delta(\epsilon)>0$ such that $\left|\phi(x)-\phi\left(x^{\prime}\right)\right| \leqq \epsilon$ if $\left|x-x^{\prime}\right| \leqq \delta(\epsilon)$, for $x, x^{\prime}$ in $I$, then we call the function $\delta(\epsilon)$ a modulus of continuity of $\phi(x)$ (in the interval $I$ ).

‡ Göttinger Nachrichten, 1914, pp. 101-109, and 1922, pp. 69-70.

§ Acta Societatis Litterarum ac Scientiarum, Szeged, vol. 1 (1923), pp. 180-186.

|| Göttinger Nachrichten, 1930, pp. 344-369 (Theorem III).

If Comptes Rendus, vol. 112 (1891), pp. 653-657.

†† These Transactions, vol. 13 (1912), pp. 109-132, and vol. 33 (1931), pp. 486-510.

$\ddagger \ddagger$ Mathematische Annalen, vol. 104 (1931), pp. 182-243 (Theorems 21, 22, 23).

$\S \S$ Cf. Göttinger Nachrichten, 1932, pp. 73-86.

III See p. 326. 
If, however, one is interested only in the existence at a given point, or only in the existence and continuity on an arc, of the $n$th derivative at the boundary, then the results of the present paper are the less restrictive.

\section{Preliminary theorems}

1. The converse of a theorem of Lindelöf. If a Jordan $\operatorname{arc} c$ has a tangent at a point $P$ and if every cord $P_{1} P_{2}$ of $c\left(P_{1} \neq P_{2}\right)$ approaches the tangent at $P$ as $P_{1}$ and $P_{2}$ approach $P$ simultaneously, we say that $c$ has an $L$-tangent at $P$. This idea was introduced by Lindelöf $\dagger$ in the statement of the following theorem, due to him: Let $w=f(z)$ be regular in the circle $|z|<1$ and let $f(z)$ map a neighborhood $\{|z-1|<r,|z|<1\}$ of $z=1$ conformally on a region bounded by a closed Jordan curve which has an $L$-tangent at the point $w_{1}=f(1)$. Then $\lim _{z \rightarrow 1} \operatorname{arc} f^{\prime}(z)$ exists for unrestricted approach in $|z|<1$.

We shall need the following converse of Lindelöf's theorem:

THEOREM I. Let $f(z)$ be regular in $|z|<1$ and let any branch of $\operatorname{arc} f^{\prime}(z)$ be harmonic in the region $\mathfrak{R}_{0}\left\{|z-1|<r_{0}<1,|z|<1\right\}$. Let $\lim _{z \rightarrow 1} \operatorname{arc} f^{\prime}(z)$ exist for unrestricted approach in $|z|<1$. Then we have

(1) $f(z)$ assumes continuous boundary values $f\left(e^{i \theta}\right)$ on an arc $\gamma$ of $|z|=1 \ddagger$ with mid-point $z=1$. Furthermore,

$$
\lim _{r \uparrow 1} f^{\prime}\left(r e^{i \theta}\right)=f^{\prime}\left(e^{i \theta}\right) \S
$$

exists almost everywhere on $\gamma$ and

$$
f\left(e^{i \theta}\right)-f(1)=\int_{0}^{\theta} f^{\prime}\left(e^{i t}\right) i e^{i t} d t,
$$

$\left|f^{\prime}\left(e^{i t}\right)\right|$ being integrable on $\gamma$ in the sense of Lebesgue.

(2) For some $r>0, w=f(z)$ is univalent in $\mathfrak{R}:\{|z-1|<r,|z| \leqq 1\}$; thus the boundary of $\mathfrak{N}$ is mapped on a closed Jordan curve $\Gamma$.

(3) $\Gamma$ has an L-tangent at the point $w_{1}=f(1)$.

The following lemma will be used in the proof.

LeMma 1. If $f(z)$ is regular in a convex region $D$ and if $\Re\left(f^{\prime}(z)\right)=u(z)>0$ in $D$, then $f(z)$ is univalent in $D . \|$

$\dagger$ Compte Rendu du 4ième Congrès des Mathématiciens Scandinaves d Stockholm (1916), pp. 89-91. The term " $L$-tangent" was introduced by A. Ostrowski, Acta Mathematica, vol. 64 (1934), pp. 81185, see p. 93.

$\ddagger$ We say that a function $f(z)$, regular in $|z|<1$, assumes continuous boundary values on an arc $\gamma$ of $|z|=1$ if there is a function $f\left(e^{i \theta}\right)$ continuous on $\gamma$ such that $\lim _{z \rightarrow e^{i \theta}} f(z)=f\left(e^{i \theta}\right)$ for unrestricted approach.

$\S$ The symbol $\alpha \uparrow a$, which was introduced by A. Ostrowski, means that $\alpha$ approaches $a$ monotonically from below.

|| See J. Wolff, Comptes Rendus, vol. 198 (1934), pp. 1209-1210. 
For, we have first

$$
f\left(z_{2}\right)-f\left(z_{1}\right)=\int_{z_{1}}^{z_{2}} f^{\prime}(\zeta) d \zeta
$$

the integral being taken along the straight line from $z_{1}$ to $z_{2}$ in $D$. Set $z_{2}=z_{1}+l e^{i \alpha}, \zeta=z_{1}+\lambda e^{i \alpha}, 0 \leqq \lambda \leqq l$. Then

$$
\left|f\left(z_{2}\right)-f\left(z_{1}\right)\right|=\left|\int_{0}^{l} f^{\prime}(\zeta) e^{i \alpha} d \lambda\right|=\left|\int_{0}^{l} f^{\prime}(\zeta) d \lambda\right| \geqq \int_{0}^{l} u(\zeta) d \lambda>0,
$$

the last integral being zero if and only if $z_{1}=z_{2}$.

Proof of the Theorem I. (1) We may assume, without loss of generality, that $\lim _{z \rightarrow 1}$ arc $f^{\prime}(z)=0$. Let $r_{1}>0$ be chosen so that $\left|\operatorname{arc} f^{\prime}(z)\right| \leqq \eta$ in $\mathfrak{R}_{1}$ : $\left\{|z-1|<r_{1},|z|<1\right\}$, where $\eta$ denotes any positive number $<(\log 2) /(4 e)$. We choose a subarc $\gamma$ of the part of $|z|=1$ belonging to the boundary of $\mathfrak{N}_{1}$ with mid-point $z=1$, and join its end points by another Jordan $\operatorname{arc} \gamma^{\prime}$ within $\mathfrak{N}_{1}$ in such a manner that $\gamma$ and $\gamma^{\prime}$ form a closed Jordan curve $C$ with continuous curvature. Let $z=\phi(\zeta)$ map the circle $|\zeta|<1$ on the interior of $C$ with $\zeta=1$ corresponding to $z=1$. The function $\phi(\zeta)$ has continuous boundary values on $|\zeta|=1$ and continuous non-vanishing first derivative in $|\zeta| \leqq 1$. $\dagger$

Since $\left|\operatorname{arc} f^{\prime}(\phi(\zeta))\right| \leqq \eta$, to every positive $p<(\log 2) /(2 e \eta)$ there corresponds a constant $K$, depending only on $p$ and $\eta$, such that

$$
\int_{0}^{2 \pi}\left|f^{\prime}\left(\phi\left(\rho e^{i \tau}\right)\right)\right|^{ \pm p} d \tau \leqq K\left|f^{\prime}(\phi(0))\right|^{ \pm p}, \text { if } 0 \leqq \rho<1 . \ddagger
$$

† See, for example, W. Seidel, Mathematische Annalen, vol. 104 (1931), p. 217, Theorem 18, and p. 226, Theorem 20, or WR, p. 433, Theorem 10, or the theorem of O. D. Kellogg quoted in the introduction.

$\ddagger$ We use the following theorem (see Göttinger Nachrichten, 1930, p. 356, Lemma 1): Let $F(z)=U(z)+i V(z)$, with $U(0)=0$, be regular in $|z|=\left|r e^{i \theta}\right|<1$ and let $|V(z)| \leqq \eta, \eta>0$. Then for any $p<(\log 2) /(2 e \eta)$ there is a constant $K(\eta, p) \geqq 1$ depending only on $\eta$ and $p$, such that

$$
\int_{0}^{2 \pi} \exp \left[p\left|F\left(r e^{i \theta}\right)\right|\right] d \theta \leqq K(\eta, p),
$$

if $0 \leqq r<1$. We apply this to $F(\zeta)=\log f^{\prime}(\phi(\zeta))-\log \left|f^{\prime}(\phi(0))\right|$ and obtain

$$
\int_{0}^{2 \pi} \exp \left[p\left|\log \frac{f^{\prime}\left(\phi\left(\rho e^{i \tau}\right)\right)}{\left|f^{\prime}(\phi(0))\right|}\right|\right] d \tau \leqq K(\eta, p) \quad(p>0)
$$

and since for any $\alpha: \exp [\Re(\alpha)] \leqq \exp [|\alpha|]$, we have

$$
\begin{aligned}
\int_{0}^{2 \pi}\left|\frac{f^{\prime}\left(\phi\left(\rho e^{i \tau}\right)\right)}{f^{\prime}(\phi(0))}\right|^{ \pm p} d \tau & =\int_{0}^{2 \pi} \exp \left[ \pm p \log \left|\frac{f^{\prime}\left(\phi\left(\rho e^{i \tau}\right)\right)}{f^{\prime}(\phi(0))}\right|\right] d \tau \\
& \leqq \int_{0}^{2 \pi} \exp \left[p\left|\log \frac{f^{\prime}\left(\phi\left(\rho e^{i \tau}\right)\right)}{\left|f^{\prime}(\phi(0))\right|}\right|\right] d \tau \leqq K(\eta, p) \text {, which gives (1.1). }
\end{aligned}
$$


As $(\log 2) /(2 e \eta)>2$, we may take $p=1$ in (1.1). Since $\left|\phi^{\prime}(\zeta)\right|$ is bounded in $|\zeta| \leqq 1:\left|\phi^{\prime}(\zeta)\right| \leqq M$, we infer from (1.1) that

$$
\int_{0}^{2 \pi}\left|f^{\prime}\left(\phi\left(\rho e^{i \tau}\right)\right)\right|\left|\phi^{\prime}\left(\rho e^{i \tau}\right)\right| d \tau \leqq M \int_{0}^{2 \pi}\left|f^{\prime}\left(\phi\left(\rho e^{i \tau}\right)\right)\right| d \tau \leqq \text { constant }
$$

for every $\rho$ in $0 \leqq \rho<1$. Therefore, as is well known, $\dagger$

$$
\lim _{\zeta \rightarrow e^{i \tau}} f^{\prime}(\phi(\zeta)) \phi^{\prime}(\zeta)=g(\tau)
$$

exists for almost every $\tau$, when $\zeta$ approaches $e^{i r}$ in any angle lying in $|\zeta|<1$ with vertex at $\zeta=e^{i \tau}$. Furthermore,

$$
f\left(\phi\left(e^{i \tau}\right)\right)-f(\phi(1))=\int_{0}^{\tau} g(\tau) i e^{i \tau} d \tau,
$$

$|g(\tau)|$ being integrable on $|\zeta|=1$. Since $\phi^{\prime}(\zeta)$ is continuous in $|\zeta| \leqq 1$ and not zero, part (1) of the conclusion then follows.

Remark. If, for any branch of $\log f^{\prime}(z)$ in $\mathfrak{N}_{0}$, we set

$$
f^{*}(z)=\int_{z_{0}}^{z} \frac{\log f^{\prime}(u) d u}{u},
$$

where the integral is taken along the straight line $z_{0} z$ in $\mathfrak{N}_{0}$ from a fixed point $z_{0}$, then $f^{*}(z)$ also assumes absolutely continuous boundary values $f^{*}\left(e^{i \theta}\right)$ on the $\operatorname{arc} \gamma$ of $|z|=1$ and

$$
f^{*}\left(e^{i \theta}\right)-f^{*}(1)=\int_{0}^{\theta} \log f^{\prime}\left(e^{i t}\right) i d t,
$$

where $\left|\log f^{\prime}\left(e^{i t}\right)\right|$ is also integrable.

For since, for any $z$ in the interior of the curve $C$ mentioned above,

$$
\left|\log f^{\prime}(z)\right| \leqq|\log | f^{\prime}(z)||+\left|\operatorname{arc} f^{\prime}(z)\right| \leqq\left|f^{\prime}(z)\right|+\frac{1}{\left|f^{\prime}(z)\right|}+\text { constant, }
$$

it follows from (1.1) for $p=1$ that the integral

$$
\int_{0}^{2 \pi}\left|\log f^{\prime}\left(\phi\left(\rho e^{i r}\right)\right)\right|\left|\phi^{\prime}\left(\rho e^{i \tau}\right)\right| \frac{d \tau}{\left|\phi\left(\rho e^{i \tau}\right)\right|}
$$

is bounded for $0 \leqq \rho<1$, from which the conclusion follows.

(2) Part (2) of the conclusion of our theorem is an immediate consequence of Lemma 1 since we have in $\mathfrak{R}_{1}\left|\operatorname{arc} f^{\prime}(z)\right| \leqq \eta<\pi / 2$ and therefore $\Re\left(f^{\prime}(z)\right)>0$.

† See, for example, F. Riesz, Mathematische Zeitschrift, vol. 18 (1923), pp. 87-95. 
(3) The existence of the $L$-tangent at $w_{1}=f(1)$ evidently follows from the following fact. Let $\epsilon>0$ be an arbitrary number. Then, for any two points $z_{1}=e^{i \theta_{1}}, z_{2}=e^{i \theta_{2}}, \theta_{1}<\theta_{2}$, in a sufficiently small neighborhood of $z=1$, we have, for a suitable branch of the argument,

$$
\left|\operatorname{arc}\left(f\left(z_{2}\right)-f\left(z_{1}\right)\right)-\frac{\pi}{2}\right| \leqq \epsilon .
$$

In order to prove this, we first note that we can choose $\delta=\delta(\epsilon)<\epsilon / 2$ such that, for $z$ belonging to $T:\{|\operatorname{arc} z| \leqq \delta(\epsilon), 0 \leqq 1-|z| \leqq \delta(\epsilon)\}$

$$
\left|\operatorname{arc} f^{\prime}(z)\right| \leqq \frac{\epsilon}{2}
$$

Let $z_{1}=e^{i \theta_{1}}, z_{2}=e^{i \theta_{2}}, \theta_{1}<\theta_{2}$, be two fixed points in $T$. According to Rolle's theorem, to each $r$ with $1-\delta(\epsilon)<r<1$, there corresponds a point $z_{0}=r e^{i \theta_{0}}$, $\theta_{1}<\theta_{0}<\theta_{2}$, such that for a suitable branch of arc $\left(f\left(r z_{2}\right)-f\left(r z_{1}\right)\right)$

$$
\operatorname{arc}\left(f\left(r z_{2}\right)-f\left(r z_{1}\right)\right)=\operatorname{arc} f^{\prime}\left(z_{0}\right)+\theta_{0}+\frac{\pi}{2} .
$$

Since $e^{i \theta_{1}}$ and $e^{i \theta_{2}}$ lie in $T,\left|\theta_{0}\right| \leqq \delta(\epsilon)<\epsilon / 2$. Therefore, since $r$ satisfies $1-\delta(\epsilon)<r<1$, it follows from (1.3) that

$$
\left|\operatorname{arc}\left(f\left(r z_{2}\right)-f\left(r z_{1}\right)\right)-\frac{\pi}{2}\right| \leqq \epsilon .
$$

Since $z_{2}, z_{1}$ are fixed, we may let $r$ approach 1 . Hence (1.2) is valid.

2. On a property of certain functions. We prove the following theorem:

THEOREM II. Let $h(z)=u(z)+i v(z)$ be regular in the circle $|z|<1, h(0)=0$, and let $\lim _{z \rightarrow 1} v(z)=v_{0}$ exist for unrestricted approach in $|z|<1$. Suppose that $\lim _{r+1} h(r)=h_{0}$ exists. Then the functions

$$
F(z)=\int_{0}^{z} e^{h(u)} d u \text { and } G(z)=\frac{1}{i} \int_{0}^{z} \frac{h(u)}{u} d u,
$$

regular in $|z|<1$, assume (absolutely) continuous boundary values on an arc $\gamma$ of $|z|=1$ with mid-point $z=1$, and have the derivatives $F^{\prime}(1)=e^{h_{0}}, G^{\prime}(1)$ $=h_{0} / i$ at $z=1$.

$\dagger$ A function $f(z)$ which is regular in $|z|<1$ and defined on $|z|=1$ in a neighborhood of a point $z=z_{1}$ of $|z|=1$ is said to be differentiable at $z_{1}$ if

exists for unrestricted approach in $|z| \leqq 1, z \neq z_{1}$.

$$
\lim _{z \rightarrow z_{1}} \frac{f(z)-f\left(z_{1}\right)}{z-z_{1}}
$$


First we see, by applying Theorem I to $F(z)$, that $F(z)$ maps the interior of a certain region $\mathfrak{R}:\{|z-1|<r,|z|<1\}$ on the interior of a closed Jordan curve $\Gamma$ which has an $L$-tangent at $w_{1}=F(1)$. Let $z=\phi(\zeta)$ be a function which maps the circle $|\zeta|<1$ on $\mathfrak{N}$ in such a manner that $\phi(1)=1$ and that the segment $-1 \leqq \zeta \leqq 1$ corresponds to the segment $1-r \leqq z \leqq 1$. Then $\phi(\zeta)$ is analytic also in a neighborhood of $\zeta=1$, and $\phi^{\prime}(1) \neq 0$. The function $e^{h(\phi(\zeta))} \phi^{\prime}(\zeta)$ tends to $e^{h_{0}} \phi^{\prime}(1)$ as $\zeta$ approaches 1 along the radius at $\zeta=1$. Therefore, $F(\phi(\zeta))$ satisfies the hypothesis of a theorem of the writer, $\dagger$ according to which

$$
\left[\frac{d F(\phi(\zeta))}{d \zeta}\right]_{\zeta=1}
$$

exists and is equal to $e^{h_{0}} \phi^{\prime}(1)$.

Hence also, for unrestricted approach in $|z| \leqq 1, z \neq 1$,

$$
\lim _{z \rightarrow 1} \frac{F(z)-F(1)}{z-1}=e^{h_{0}} \text {. }
$$

We shall now use (2.1) to prove the conclusion regarding $G(z)$.

According to the Remark above, $G(z)$ is continuous on an arc $\gamma$ of $|z|=1$ with mid-point $z=1$, and we have, for $e^{i \theta}$ on $\gamma$,

$$
G\left(e^{i \theta}\right)-G(1)=\int_{0}^{\theta} h\left(e^{i t}\right) d t,
$$

.where

$$
h\left(e^{i t}\right)=u\left(e^{i t}\right)+i v\left(e^{i t}\right)=\lim _{\uparrow \uparrow 1} h\left(r e^{i t}\right)
$$

exists for almost every $e^{i t}$ on $\gamma$ and $\left|h\left(e^{i t}\right)\right|$ is integrable along $\gamma$. It is sufficient to prove the conclusion, namely

$$
\lim _{z \rightarrow 1} \frac{G(z)-G(1)}{z-1}=\frac{1}{i} h_{0},
$$

only for the case that $z$ approaches 1 along $|z|=1(z \neq 1)$, that is, to prove that

$$
\lim _{\theta \rightarrow 0} \frac{1}{\theta} \int_{0}^{\theta}\left[h\left(e^{i t}\right)-h_{0}\right] d t=0 .
$$

$\dagger$ If $w=f(z)$ maps the circle $|z|<1$ on the interior of a closed Jordan curve $C$ in such a manner that $z=1$ corresponds to $w_{1}$ on $C$, if $C$ has an $L$-tangent in $w_{1}$ and if $\lim _{r+1} f^{\prime}(r)$ exists, then $f(z)$ is differentiable at $z=1$ and $f^{\prime}(1)=\lim _{r+1} f^{\prime}(r)$. This is a special case of a theorem in WR, p. 376 (Theorem 3). Compare also the paper of the writer in Compositio Mathematica, vol. 1 (1935), p. 320. 
For, since (2.2) implies that $\Phi(z) \equiv(G(z)-G(1)) /(z-1)$ is bounded on $\gamma$, and since $\Phi(z)=O(1 /|1-z|)$ in a region $\mathfrak{R}_{1}\left\{|z-1| \leqq r_{1},|z|<1\right\}$ it follows from a well known theorem of Phragmén-Lindelöf that $\Phi(z)$ is bounded in $\mathfrak{N}_{1}$. Hence, according to a theorem of Lindelöf, it follows from (2.2) that $\lim _{z \rightarrow 1} \Phi(z)=h_{0} / i$ for unrestricted approach in $|z|<1$.

As $v\left(e^{i \theta}\right)$, defined for almost every $e^{\imath \theta}$ on $\gamma$, is continuous at $\theta=0$ we have

$$
\lim _{\theta \rightarrow 0} \frac{1}{\theta} \int_{0}^{\theta}\left[v\left(e^{i t}\right)-v_{0}\right] d t=0 \text {. }
$$

Therefore, (2.2) is equivalent to

$$
\lim _{\theta \rightarrow 0} \frac{1}{\theta} \int_{0}^{\theta}\left[u\left(e^{i t}\right)-u_{0}\right] d t=0 .
$$

In order to prove (2.3), we first note that we may infer from (2.1), with the help of the relation

that

$$
\int_{e^{i \theta}}^{1}\left[e^{h(\zeta)}-\epsilon^{h_{0}}\right] d \zeta=-i e^{h_{0}} \int_{0}^{\theta}\left[e^{h\left(e^{i t}\right)-h_{0}}-1\right] e^{i t} d t \quad(|\zeta|=1)
$$

$$
\lim _{\theta \rightarrow 0} \frac{1}{\theta} \int_{0}^{\theta} e^{\left[u\left(e^{i t}\right)-u_{0}\right]+i\left(v\left(e^{i t}\right)-v_{0}\right]} e^{i t} d t=1 .
$$

Since, according to Theorem I, (1), $\left|e^{h\left(e^{i t}\right)}\right|=e^{u\left(e^{i t}\right)}$ and hence $e^{u\left(e^{i t}\right)-u_{0}}$ is $L$-integrable along $\gamma$, we have, $|\theta|<\pi$,

$$
\begin{aligned}
\left|\frac{1}{\theta} \int_{0}^{\theta} e^{\left[u\left(e^{i t}\right)-u_{0}\right]+i\left[v\left(e^{i t}\right)-v_{0}\right]}\left(e^{i t}-1\right) d t\right| & \leqq \frac{1}{\theta} \int_{0}^{\theta} e^{u(e i t)-u_{0}}\left|e^{i t}-1\right| d t \\
& \leqq \frac{2|\sin (\theta / 2)|}{\theta} \int_{0}^{\theta} e^{u(e i t)-u_{0} d t},
\end{aligned}
$$

which approaches 0 with $\theta$. Therefore it follows from (2.4) that

$$
\lim _{\theta \rightarrow 0} \frac{1}{\theta} \int_{0}^{\theta} e^{\left[u(e i t)-u_{0}\right]+i\left[v(e i t)-v_{0}\right]} d t=1 .
$$

Because of the continuity of $v\left(e^{i \theta}\right)$ at $\theta=0$ it follows from (2.5) that

$$
\lim _{\theta \rightarrow 0} \frac{1}{\theta} \int_{0}^{\theta}\left[e^{u(e i t)-u_{0}}-1\right] d t=0 .
$$

Now, since $-h(z)$ satisfies the hypotheses of the theorem, if $h(z)$ satisfies them, (2.1) remains true when we replace $F(z)$ by $F_{1}(z)=\int_{0}^{z} e^{-h(u)} d u$ and $e^{h_{0}}$ by $e^{-h_{0}}$. Therefore we obtain, by the method used to establish (2.6), 


$$
\lim _{\theta \rightarrow 0} \frac{1}{\theta} \int_{0}^{\theta}\left[e^{-\left(u\left(e^{i t}\right)-u_{0}\right)}-1\right] d t=0 .
$$

With the aid of the inequality

$$
\alpha^{2} \leqq e^{\alpha}+e^{-\alpha}-2=e^{\alpha}-1+e^{-\alpha}-1,
$$

which holds for every real $\alpha$, we obtain from (2.6) and (2.7):

Hence

$$
\begin{aligned}
\frac{1}{\theta} \int_{0}^{\theta}\left[u\left(e^{i t}\right)-u_{0}\right]^{2} d t \leqq & \frac{1}{\theta} \int_{0}^{\theta}\left[e^{\left(u\left(e^{i t}\right)-u_{0}\right)}-1\right] d t \\
& +\frac{1}{\theta} \int_{0}^{\theta}\left[e^{-\left(u\left(\theta^{i t}\right)-u_{0}\right)}-1\right] d t .
\end{aligned}
$$

$$
\lim _{\theta \rightarrow 0} \frac{1}{\theta} \int_{0}^{\theta}\left[u\left(e^{i t}\right)-u_{0}\right]^{2} d t=0 .
$$

From Schwarz's inequality it then follows that

$$
\left(\frac{1}{\theta} \int_{0}^{\theta}\left[u\left(e^{i t}\right)-u_{0}\right] d t\right)^{2} \leqq \frac{1}{\theta} \int_{0}^{\theta}\left[u\left(e^{i t}\right)-u_{0}\right]^{2} d t
$$

which proves (2.3). Therefore the theorem is true.

II. EXISTENCE AND CONTINUITY OF THE $n$ TH DERIVATIVE OF THE MAPPING FUNCTION ON THE BOUNDARY

3. Lemmas. Let $V(s)$ be defined and continuous in the interval $I$ : $A \leqq s \leqq B$, and, for an $s$ in $I$ and an $a>0$, let the integral

$$
\int_{0}^{a}|V(s+t)+V(s-t)-2 V(s)| \frac{d t}{t^{2}}
$$

exist. We shall make a few remarks about this integral which we shall use later.

(1) If $V^{\prime}(s)$ exists and is continuous in $I$, and if

$$
\int_{0}^{a}\left|V^{\prime}(s+t)-V^{\prime}(s-t)\right| \frac{d t}{t}
$$

exists for an $s$ in $I$, then also (3.1) exists and we have

$$
\begin{gathered}
\int_{0}^{a}|V(s+t)+V(s-t)-2 V(s)| \frac{d t}{t^{2}} \\
\leqq \int_{0}^{a}\left|V^{\prime}(s+t)-V^{\prime}(s-t)\right| \frac{d t}{t} .
\end{gathered}
$$


For, if we set $\psi(\sigma)=V^{\prime}(s+\sigma)-V^{\prime}(s-\sigma)$ and if $0<\epsilon<a$, we have

$$
\begin{aligned}
\int_{a}^{a}|V(s+t)+V(s-t)-2 V(s)| \frac{d t}{t^{2}} & \leqq \int_{0}^{a} \frac{d t}{t^{2}} \int_{0}^{t}|\psi(\sigma)| d \sigma \\
& =\iint_{T_{e}} \frac{|\psi(\sigma)|}{t^{2}} d \sigma d t
\end{aligned}
$$

the double integral being taken over the quadrilateral $T_{e}$ with vertices $\sigma=0, t=\epsilon ; \sigma=\epsilon, t=\epsilon ; \sigma=a, t=a ; \sigma=0, t=a$. Now we observe that

$$
\begin{aligned}
\iint_{T_{\epsilon}} \frac{|\psi(\sigma)|}{t^{2}} d \sigma d t & =\int_{e}^{a}|\psi(\sigma)| \int_{\sigma}^{a} \frac{d t}{t^{2}} d \sigma+\int_{0}^{e}|\psi(\sigma)| \int_{0}^{a} \frac{d t}{t^{2}} d \sigma \\
& \leqq \int_{0}^{a} \frac{|\psi(\sigma)|}{\sigma} d \sigma,
\end{aligned}
$$

and letting $\epsilon$ approach zero, we see that the result is true.

(1a) If $V^{\prime}(s)$ satisfies a Hölder condition at $s=s_{1}$ :

$$
\left|V^{\prime}\left(s_{1}+t\right)-V^{\prime}\left(s_{1}\right)\right| \leqq H|t|^{\beta}, \quad 0<\beta \leqq 1,
$$

then, because of (3.2),

$$
\int_{0}^{a}\left|V\left(s_{1}+t\right)+V\left(s_{1}-t\right)-2 V\left(s_{1}\right)\right| \frac{d t}{t^{2}} \leqq \frac{2^{\beta} H}{\beta} a^{\beta} .
$$

(2) Sum and product. We shall sometimes use the notation

$$
\Delta_{t}^{(1)} V(s) \equiv V(s+t)-V(s), \quad \Delta_{t}^{(2)} V(s) \equiv V(s+t)+V(s-t)-2 V(s) .
$$

If $V_{1}(s)$ and $V_{2}(s)$ are defined and continuous in $I$, we have

$$
\begin{aligned}
\int_{0}^{a}\left|\Delta_{t}^{(2)}\left[V_{1}(s)+V_{2}(s)\right]\right| \frac{d t}{t^{2}} \leqq & \int_{0}^{a}\left|\Delta_{t}^{(2)} V_{1}(s)\right| \frac{d t}{t^{2}} \\
& +\int_{0}^{a}\left|\Delta_{t}^{(2)} V_{2}(s)\right| \frac{d t}{t^{2}}
\end{aligned}
$$

provided both integrals on the right exist.

If, throughout $I,\left|V_{1}(s)\right| \leqq M_{1},\left|V_{2}(s)\right| \leqq M_{2}$, if, for a certain value $s=s_{0}$ in $A<s<B,(3.1)$ exists for $V(s)=V_{1}(s), V(s)=V_{2}(s)$, and if

$$
\left|\Delta_{t}^{(1)} V_{1}\left(s_{0}\right)\right| \leqq K_{1}|t|, \quad\left|\Delta_{t}^{(1)} V_{2}\left(s_{0}\right)\right| \leqq K_{2}|t|
$$

( $M_{1}, M_{2}, K_{1}, K_{2}$ being constants), we have 


$$
\begin{aligned}
\int_{0}^{a}\left|\Delta_{t}^{(2)}\left[V_{1}\left(s_{0}\right) V_{2}\left(s_{0}\right)\right]\right| \frac{d t}{t^{2}} \leqq & M_{1} \int_{0}^{a}\left|\Delta_{t}^{(2)} V_{2}\left(s_{0}\right)\right| \frac{d t}{t^{2}} \\
& +M_{2} \int_{0}^{a}\left|\Delta_{t}^{(2)} V_{1}\left(s_{0}\right)\right| \frac{d t}{t^{2}}+2 K_{1} K_{2} a .
\end{aligned}
$$

For

$$
\begin{aligned}
\Delta_{t}^{(2)}\left[V_{1}\left(s_{0}\right) V_{2}\left(s_{0}\right)\right]= & V_{1}\left(s_{0}+t\right) V_{2}\left(s_{0}+t\right)+V_{1}\left(s_{0}-t\right) V_{2}\left(s_{0}-t\right) \\
& -2 V_{1}\left(s_{0}\right) V_{2}\left(s_{0}\right) \\
= & V_{1}\left(s_{0}+t\right)\left[V_{2}\left(s_{0}+t\right)+V_{2}\left(s_{0}-t\right)-2 V_{2}\left(s_{0}\right)\right] \\
& +V_{2}\left(s_{0}-t\right)\left[V_{1}\left(s_{0}+t\right)+V_{1}\left(s_{0}-t\right)-2 V_{1}\left(s_{0}\right)\right] \\
& -2 V_{1}\left(s_{0}+t\right) V_{2}\left(s_{0}-t\right)+2 V_{2}\left(s_{0}\right) V_{1}\left(s_{0}+t\right) \\
& +2 V_{1}\left(s_{0}\right) V_{2}\left(s_{0}-t\right)-2 V_{1}\left(s_{0}\right) V_{2}\left(s_{0}\right) \\
= & V_{1}\left(s_{0}+t\right) \Delta_{t}^{(2)} V_{2}\left(s_{0}\right)+V_{2}\left(s_{0}+t\right) \Delta_{t}^{(2)} V_{1}\left(s_{0}\right) \\
& -2\left[V_{1}\left(s_{0}+t\right)-V_{1}\left(s_{0}\right)\right]\left[V_{2}\left(s_{0}-t\right)-V_{2}\left(s_{0}\right)\right] .
\end{aligned}
$$

By multiplying this by $1 / t^{2}$ and integrating over $0 \cdots a$, we obtain a relation from which (3.5) follows at once.

(3) Change of variables. Let (3.1) converge at $s=s_{1}, A<s_{1}<B$, and let

$$
\left|V\left(s^{\prime}\right)-V\left(s^{\prime \prime}\right)\right| \leqq K\left|s^{\prime}-s^{\prime \prime}\right|
$$

for $s^{\prime}, s^{\prime \prime}$ in $I$. Let $s=s(\theta), A \leqq s(\theta) \leqq B$, be defined in the interval $I^{*}$, with continuous positive first derivative. Thus there are two constants $\mu_{1}, \mu_{2}$ such that for $\theta^{\prime}$ and $\theta^{\prime \prime}$ in $I^{*}$,

$$
0<\mu_{1} \leqq \frac{s\left(\theta^{\prime}\right)-s\left(\theta^{\prime \prime}\right)}{\theta^{\prime}-\theta^{\prime \prime}} \leqq \mu_{2} .
$$

Suppose that $s_{1}=s\left(\theta_{1}\right)$ and that for an $\alpha>0$

$$
\int_{0}^{\alpha}\left|s\left(\theta_{1}+\tau\right)+s\left(\theta_{1}-\tau\right)-2 s\left(\theta_{1}\right)\right| \frac{d \tau}{\tau^{2}}
$$

exists. Then, if we denote $V(s(\theta))$ by $V^{*}(\theta)$,

$$
\int_{0}^{\alpha}\left|\Delta_{\tau}^{(2)} V^{*}\left(\theta_{1}\right)\right| \frac{d \tau}{\tau^{2}}
$$

also exists, and we have, for $a=s\left(\theta_{1}+\alpha\right)-s\left(\theta_{1}\right)$,

$$
\int_{0}^{\alpha}\left|\Delta_{\tau}^{(2)} V^{*}\left(\theta_{1}\right)\right| \frac{d \tau}{\tau^{2}} \leqq \frac{\mu_{2}^{2}}{\mu_{1}} \int_{0}^{a}\left|\Delta_{t}^{(2)} V\left(s_{1}\right)\right| \frac{d t}{t^{2}}+K \int_{0}^{\alpha}\left|\Delta_{\tau}^{(2)} s\left(\theta_{1}\right)\right| \frac{d \tau}{\tau^{2}} .
$$


For if, for sufficiently small $|\tau|$, we set $s\left(\theta_{1}+\tau\right)=s_{1}+t, s\left(\theta_{1}-\tau\right)=s_{1}-t^{\prime}$, we have

$$
\Delta_{\tau}^{(2)} V^{*}\left(\theta_{1}\right)=\Delta_{t}^{(2)} V\left(s_{1}\right)+V\left(s_{1}-t^{\prime}\right)-V\left(s_{1}-t\right) .
$$

It follows from (3.6) that

$$
\left|V\left(s_{1}-t^{\prime}\right)-V\left(s_{1}-t\right)\right| \leqq K\left|t-t^{\prime}\right|=K\left|s\left(\theta_{1}+\tau\right)+s\left(\theta_{1}-\tau\right)-2 s\left(\theta_{1}\right)\right| \text {. }
$$

If we denote by $\theta(s)$ the inverse function of $s(\theta)$, we obtain

$$
\begin{aligned}
\int_{0}^{\alpha}\left|\Delta_{\tau}^{(2)} V^{*}\left(\theta_{1}\right)\right| \frac{d \tau}{\tau^{2}} & \leqq \int_{0}^{a}\left|\Delta_{t}^{(2)} V\left(s_{1}\right)\right|\left(\frac{t}{\tau}\right)^{2} \frac{d \theta\left(s_{1}+t\right)}{d t} \frac{d t}{t^{2}} \\
& +K \int_{0}^{\alpha}\left|\Delta_{\tau}^{(2)} s\left(\theta_{1}\right)\right| \frac{d \tau}{\tau^{2}}
\end{aligned}
$$

from which (3.8) follows with the help of (3.7).

We shall also have to use the following simple lemmas.

LEMMA 2. Let $f(t)$ be continuous for $A \leqq t \leqq B, 0<\lambda \leqq B-A \leqq \Lambda$, and let $\delta(\epsilon)$ be a modulus of continuity of $f(t)$. Suppose $\left|\int_{A}^{B} f(t) d t\right| \leqq m, m \geqq 0$. Then there is a number $M \geqq 1$, which depends only on $m, \lambda, \Lambda$ and the function $\delta(\epsilon)$, but not otherwise on $f(t)$, such that in $A \leqq t \leqq B:|f(t)| \leqq M$.

We may omit the simple proof of this lemma. We shall need the following corollary.

LEMMA 3. Let $f(t)$ have in $A \leqq t \leqq B$, with $0<\lambda \leqq B-A \leqq \Lambda$, a continuous nth derivative and let $\delta(\epsilon)$ be a modulus of continuity of $f^{(n)}(t)$. Suppose that $\left|\int_{A}^{B} f^{(\nu)}(t) d t\right| \leqq m, m \geqq 0, \nu=1,2, \cdots, n$. Then there exists $a K \geqq 1$, depending only on $\lambda, \Lambda, m$, and the function $\delta(\epsilon)$, such that, in $A \leqq t \leqq B,\left|f^{(v)}(t)\right| \leqq K$, $\nu=1,2, \cdots, n$.

First, according to Lemma 2 , there is an $M_{n} \geqq 1$, depending only on $\lambda, \Lambda$, $m$ and $\delta(\epsilon)$, such that $\left|f^{(n)}(t)\right| \leqq M_{n}$ in $A \leqq t \leqq B$. Hence

$$
\left|f^{(n-1)}\left(t_{1}\right)-f^{(n-1)}\left(t_{2}\right)\right| \leqq M_{n}\left|t_{1}-t_{2}\right| \text {. }
$$

Therefore, we may apply Lemma 2 to $f^{(n-1)}(t)$ with the modulus of continuity $\delta^{*}(\epsilon)=\epsilon / M_{n}$. Thus we obtain an $M_{n-1}$, which also depends only on $\lambda, \Lambda, m$, and $\delta(\epsilon)$, such that $\left|f^{(n-1)}(t)\right| \leqq M_{n-1}$ in $A \leqq t \leqq B$. By applying the same method to $f^{(n-2)}(t)$ and $f^{(n-1)}(t), f^{(n-3)}(t)$ and $f^{(n-2)}(t)$, and so on, the proof is easily completed.

4. Radial boundary values of the derivatives of analytic functions. We prove 
LEMMA 4. Let $F(z)=u(z)+i v(z)$ be regular in $|z|<1$ and let $v(z)$ be continuous in $|z| \leqq 1$. Set $v\left(e^{i \theta}\right)=V(\theta)$.

(a) If, for a certain value of $\theta, V^{\prime}(\theta)$ and the integral

$$
\int_{0}^{a}|V(\theta+\tau)+V(\theta-\tau)-2 V(\theta)| \frac{d \tau}{\sin ^{2}(\tau / 2)}, \quad 0<a<\pi
$$

exist, then also $\lim _{r+1} F^{\prime}\left(r e^{i \theta}\right)$ exists and

$$
\begin{aligned}
& i e^{i \theta} \lim _{r+1} F^{\prime}\left(r e^{i \theta}\right) \\
& \quad=\frac{1}{4 \pi} \int_{0}^{\pi}\{V(\theta+\tau)+V(\theta-\tau)-2 V(\theta)\} \frac{d \tau}{\sin ^{2}(\tau / 2)}+i V^{\prime}(\theta) . \dagger
\end{aligned}
$$

(b) If $V^{\prime}(\theta)$ exists and is continuous on the arc $\gamma$ : $\theta_{1} \leqq \theta \leqq \theta_{2}\left(0<\theta_{2}-\theta_{1} \leqq 2 \pi\right)$ of $|z|=1$ and if (4.1) exists for every $\theta$ on $\gamma$ and approaches zero uniformly with $a$ on $\gamma$, then $F^{\prime}(z)$ assumes continuous boundary values on the open arc $\gamma$.

(c) Suppose that the hypotheses of (b) are satisfied for $\gamma \equiv\{0 \leqq \theta \leqq 2 \pi\}$ and that $V^{\prime}(0)=V^{\prime}(2 \pi)$. Suppose $\delta(\xi)$ is a modulus of continuity of $V^{\prime}(\theta)$, and $\eta(\xi)$ is a modulus of convergence $\ddagger$ of (4.1). Then also $F^{\prime}(z)$ assumes continuous boundary values $F^{\prime}\left(e^{i \theta}\right)$ on $|z|=1$ and the modulus of continuity of $F^{\prime}\left(e^{i \theta}\right), \Delta(\epsilon)$, depends only on $\epsilon$, and the functions $\delta(\xi)$ and $\eta(\xi)$. Furthermore, there is an upper bound for $\left|F^{\prime}\left(e^{i \theta}\right)\right|$, also depending only on the functions $\delta(\xi)$ and $\eta(\xi)$.

By using Poisson's formula

† Part (a) of this lemma has already been used by the author in WR, pp. 407 and 424 . A theorem of similar nature was obtained by A. Plessner, Zur Theorie der konjugierten trigonometrischen Reihen, Dissertation, Giessen, 1923, p. 2. The corresponding generalization of condition (4.1) for functions harmonic within a sphere has been used by $\mathrm{O}$. D. Kellogg, in the second paper mentioned in footnote t† on p. 311.

$\ddagger$ We call a function $\eta(\xi)>0$ defined for $\xi>0$ a modulus of convergence of the integral (4.1) if for every $\xi>0$, over all of $|z|=1$, the integral (4.1) is $\leqq \xi$, provided that $0<a \leqq \eta(\xi)$.

Since $\sin (\tau / 2) \leqq \tau / 2$ and, for $0 \leqq \tau \leqq \pi, \sin (\tau / 2) \geqq \tau / \pi$, we have

$$
\pi^{2} \int_{0}^{a}\left|\Delta_{\tau}^{(2)} V(\theta)\right| \frac{d \tau}{\tau^{2}} \geqq \int_{0}^{a}\left|\Delta_{\tau}^{(2)} V(\theta)\right| \frac{d \tau}{\sin ^{2}(\tau / 2)} \geqq 4 \int_{0}^{a}\left|\Delta_{\tau}^{(2)} V(\theta)\right| \frac{d \tau}{\tau^{2}}
$$

Hence, if $\eta(\xi)$ is a modulus of convergence for

then $\eta(\xi)$ is also one for

$$
\begin{gathered}
\int_{0}^{a}\left|\Delta_{\tau}^{(2)} V(\theta)\right| \frac{d \tau}{\sin ^{2}(\tau / 2)} \\
\int_{0}^{a}\left|\Delta_{\tau}^{(2)} V(\theta)\right| \frac{d \tau}{\tau^{2}} .
\end{gathered}
$$

Conversely, if $\eta(\xi)$ is a modulus of convergence for the second integral, $\eta\left(\xi / \pi^{2}\right)$ is one for the first one. 


$$
\begin{gathered}
v\left(r e^{i \theta}\right)=\frac{1}{2 \pi} \int_{-\pi}^{\pi} V(\phi) K(r, \phi-\theta) d \phi=\frac{1}{2 \pi} \int_{\theta-\pi}^{\theta+\pi} V(\phi) K(r, \phi-\theta) d \phi \\
\left(K(r, \alpha)=\frac{1-r^{2}}{1+r^{2}-2 r \cos \alpha}=\frac{1-r^{2}}{(1-r)^{2}+4 r \sin ^{2}(\alpha / 2)}\right),
\end{gathered}
$$

which holds for $0 \leqq r<1$, we obtain by setting $\phi-\theta=\tau$

$$
\begin{aligned}
v\left(r e^{i \theta}\right) & =\frac{1}{2 \pi} \int_{-\pi}^{\pi} V(\theta+\tau) K(r, \tau) d \tau=\frac{1}{2 \pi} \int_{0}^{\pi}\{V(\theta+\tau)+V(\theta-\tau)\} K(r, \tau) d \tau \\
& =\frac{1}{2 \pi} \int_{0}^{\pi}\{V(\theta+\tau)+V(\theta-\tau)-2 V(\theta)\} K(r, \tau) d \tau+V(\theta) .
\end{aligned}
$$

Hence

$$
\begin{aligned}
\frac{\partial v\left(r e^{i \theta}\right)}{\partial r}= & \frac{1}{\pi} \int_{0}^{\pi}\{V(\theta+\tau)+V(\theta-\tau)-2 V(\theta)\} \\
& \frac{(1-r)^{2}-2\left(1+r^{2}\right) \sin ^{2}(\tau / 2)}{\left[(1-r)^{2}+4 r \sin ^{2}(\tau / 2)\right]^{2}} d \tau .
\end{aligned}
$$

The expression

$$
\frac{(1-r)^{2}-2\left(1+r^{2}\right) \sin ^{2}(\tau / 2)}{\left[(1-r)^{2}+4 r \sin ^{2}(\tau / 2)\right]^{2}} \sin ^{2}(\tau / 2)
$$

is uniformly bounded for $\frac{1}{2} \leqq r<1$ and every real $\tau$. Furthermore, it tends to $-\frac{1}{2}$ uniformly in the interval $\delta \leqq \tau \leqq \pi$ for any fixed positive $\delta$ as $r$ approaches 1. Since the integral (4.1) approaches zero with $a$, we therefore see that

$$
r \frac{\partial v\left(r e^{i \theta}\right)}{\partial r}=-\frac{\partial u\left(r e^{i \theta}\right)}{\partial \theta} \rightarrow-\frac{1}{4 \pi} \int_{0}^{\pi} \frac{\{V(\theta+\tau)+V(\theta-\tau)-2 V(\theta)\}}{\sin ^{2}(\tau / 2)} d \tau
$$

as $r$ approaches 1 , and also that this convergence is uniform in any interval $\theta_{1} \leqq \theta \leqq \theta_{2}$ in which (4.1) tends uniformly to zero with $a$. Furthermore, according to a theorem of Fatou,

$$
\lim _{r+1} \frac{\partial v\left(r e^{i \theta}\right)}{\partial \theta}=V^{\prime}(\theta)
$$

for every $\theta$ for which

$$
V^{\prime}(\theta)=\frac{d V(\theta)}{d \theta}
$$

exists, and the convergence is uniform in $\theta_{1} \leqq \theta \leqq \theta_{2}$, if $V^{\prime}(\theta)$ is continuous there. This establishes parts (a) and (b) of the theorem. 
It follows immediately from (b) that, under the hypothesis of part (c), $F^{\prime}(z)$ assumes continuous boundary values on $|z|=1$. To prove the remaining conclusion in (c), it is evidently sufficient to prove the result for the function $i e^{i \theta} F^{\prime}\left(e^{i \theta}\right)$ instead of $F^{\prime}\left(e^{i \theta}\right)$.

As far as the modulus of continuity of $i e^{i \theta} F^{\prime}\left(e^{i \theta}\right)$ is concerned, it remains only to show that the modulus of continuity of its real part depends only on $\epsilon$ and on the functions $\delta(\xi)$ and $\eta(\xi)$. In order to demonstrate this we first note that, since $\int_{0}^{2 \pi} V^{\prime}(\theta) d \theta=0$, according to Lemma 2 there is an $M \geqq 1$, depending only on $\delta(\xi)$, such that $\left|V^{\prime}(\theta)\right| \leqq M$. Therefore $\left|V\left(\theta_{1}\right)-V\left(\theta_{2}\right)\right|$ $\leqq M\left|\theta_{1}-\theta_{2}\right|$. Let $\epsilon>0$ be an arbitrarily small number. Then we have

$$
\begin{aligned}
&\left|\int_{0}^{\pi} \frac{\left\{\Delta_{\tau}^{(2)} V\left(\theta_{1}\right)-\Delta_{\tau}^{(2)} V\left(\theta_{2}\right)\right\} d \tau}{\sin ^{2}(\tau / 2)}\right| \leqq \int_{0}^{\eta(\epsilon / 3)} \frac{\left|\Delta_{\tau}^{(2)} V\left(\theta_{1}\right)\right| d \tau}{\sin ^{2}(\tau / 2)} \\
&+\int_{0}^{\eta(\epsilon / 3)} \frac{\left|\Delta_{\tau}^{(2)} V\left(\theta_{2}\right)\right| d \tau}{\sin ^{2}(\tau / 2)}+\int_{\eta(\epsilon / 3)}^{\pi} \frac{\left|\Delta_{\tau}^{(2)}\left\{V\left(\theta_{1}\right)-V\left(\theta_{2}\right)\right\}\right| d \tau}{\sin ^{2}(\tau / 2)} \\
& \leqq \\
& \qquad \frac{2 \epsilon}{3}+\frac{4 M \pi\left|\theta_{1}-\theta_{2}\right|}{\sin ^{2}\left(\frac{1}{2} \eta(\epsilon / 3)\right)} \leqq \epsilon,
\end{aligned}
$$

provided that

Since

$$
\left|\theta_{1}-\theta_{2}\right| \leqq \frac{\epsilon}{12 M \pi} \sin ^{2}\left(\frac{1}{2} \eta(\epsilon / 3)\right)
$$

$$
\int_{0}^{2 \pi} \frac{d F\left(e^{i \theta}\right)}{d \theta} d \theta=\int_{0}^{2 \pi} i e^{i \theta} F^{\prime}\left(e^{i \theta}\right) d \theta=0
$$

it follows from Lemma 2 that there is an $N \geqq 1$, depending only on the modulus of continuity of $i e^{i \theta} F^{\prime}\left(e^{i \theta}\right)$, such that $\left|i e^{i \theta} F^{\prime}\left(e^{i \theta}\right)\right|=\left|F^{\prime}\left(e^{i \theta}\right)\right| \leqq N$ for all $\theta$. This completes the proof.

The following lemma generalizes Lemma 4 (a) and (b).

LEMMA 5. Let $F(z)=u(z)+i v(z)$ be regular in $|z|<1$ and let $v(z)$ assume continuous boundary values $V(\theta)$ on the arc $\gamma: \theta_{1} \leqq \theta \leqq \theta_{2}$ of $|z|=1$.

(a) If at $\theta=\theta_{0}=\left(\theta_{1}+\theta_{2}\right) / 2, V^{\prime}(\theta)$ exists and the integral (4.1) converges for an $a>0$, then $\lim _{r+1} F^{\prime}\left(r e^{i \theta}\right)$ exists.

(b) If $V^{\prime}(\theta)$ is continuous over all of $\gamma$ and if (4.1) exists at each point of a subarc $\gamma^{\prime}: \theta_{1}<\theta_{1}{ }^{\prime} \leqq \theta \leqq \theta_{2}{ }^{\prime}<\theta_{2}$ of $\gamma$ and approaches zero uniformly with $a$ on $\gamma^{\prime}$, then $F^{\prime}(z)$ assumes continuous boundary values on the open arc $\gamma^{\prime}$. 
Let $\Gamma$ denote the closed Jordan curve formed by $\gamma$ and the part within $|z| \leqq 1$ of the circle with center $z=e^{i \theta_{0}}$ and through the end points of $\gamma . \Gamma$ is symmetric in the diameter of the circle $|z|=1$ through $z=e^{i \theta}$. Let $z=g(\zeta)$ map the circle $|\zeta|<1$ on the interior of $\Gamma$ in such a manner that $g(\dot{1})=e^{i \theta} 0$ and that the segment $-1<\zeta<1$ corresponds to the part within $\Gamma$ of the diameter of $|z|=1$ through $e^{i \theta}$. Let $\gamma^{*}$ be the arc of $|\zeta|=1$ to which $\gamma$ corresponds. The function $g(\zeta)$ is also regular on the open arc $\gamma^{*}$ and $g^{\prime}(\zeta)$ is not zero there.

Part (a) of our lemma follows immediately from Lemma 4 (a) applied to $F(g(\zeta))$. The function $F(g(\zeta))$ satisfies its hypotheses: for, first

$$
\left(\frac{\partial v\left(g\left(e^{i \phi}\right)\right)}{\partial \phi}\right)_{\phi=0}=V^{\prime}\left(\theta_{0}\right)\left|g^{\prime}(1)\right| \text { exists, }
$$

and secondly, because of the symmetry of $\Gamma$ mentioned above, we have, if we set $g\left(e^{i \psi}\right)=e^{i\left(\theta_{0}+r\right)}$ for sufficiently small $\psi>0, g\left(e^{-i \psi}\right)=e^{i\left(\theta_{0}-r\right)}$, and hence,

$$
\begin{aligned}
& \int_{0}^{\alpha}\left|v\left(g\left(e^{i \psi}\right)\right)+v\left(g\left(e^{-i \psi}\right)\right)-2 v(g(1))\right| \frac{d \psi}{\psi^{2}} \\
& \quad=\int_{0}^{a}\left|V\left(\theta_{0}+\tau\right)+V\left(\theta_{0}-\tau\right)-2 V\left(\theta_{0}\right)\right|\left(\frac{\tau}{\psi}\right)^{2} \frac{1}{\left|g^{\prime}\left(e^{i \psi}\right)\right|} \frac{d \tau}{\tau^{2}} \\
& \quad \leqq \text { constant } \cdot \int_{0}^{a}\left|V\left(\theta_{0}+\tau\right)+V\left(\theta_{0}-\tau\right)-2 V\left(\theta_{0}\right)\right| \frac{d \tau}{\tau^{2}},
\end{aligned}
$$

where $\alpha$ is defined by $g\left(e^{i \alpha}\right)=e^{i\left(\theta_{0}+a\right)}$.

Part (b) follows from Lemma 4 (b) applied to $F(g(\zeta))$, since in this case $\partial v\left(g\left(e^{i \phi}\right)\right) / \partial \phi=V^{\prime}(\theta)\left|g^{\prime}\left(e^{i \phi}\right)\right|$ is continuous on the open arc $\gamma^{*}$, and since also the second hypothesis of Lemma 4 (b) is satisfied, as may be easily seen by use of $\S 3,(3)$.

5. Boundary values of the $n$th derivative of the mapping function. Let $c$ be a rectifiable Jordan arc with continuously turning tangent and let $\Theta(s)$ be an angle from the direction of the positive axis of reals to the tangent line, where $s$ denotes the arc length. We define $\Theta(s)$ first at an arbitrary point of $c$ and then at the other points so that $\Theta(s)$ varies continuously with $s$. If $\kappa^{(n)}(s)=d^{n} \Theta(s) / d s^{n}$ exists at $s=s_{1}$ we call $\kappa^{(n)}\left(s_{1}\right)$ the curvature of order $n$ of $c$ at $s_{1} ; \kappa^{(0)}(s)$ is understood to be $\Theta(s)$. If $\kappa^{(n-1)}(s)$ exists in a neighborhood of $s=s_{1}$ and if

$$
\lim \frac{\kappa^{(n-1)}(s)-\kappa^{(n-1)}\left(s^{\prime}\right)}{s-s^{\prime}}
$$

exists when $s$ and $s^{\prime}\left(s \neq s^{\prime}\right)$ approach $s_{1}$ simultaneously, we say that $c$ has an L-curvature of order $n$ at $s_{1}$. 
TheOREM III. Let $R$ be a simply connected region. Let the boundary of $R$ contain a free Jordan arc c. $\dagger$ Suppose $w=f(z)$ is a function which maps the circle $|z|<1$ on $R$.

(a) If in a neighborhood of a point $w_{1}\left(s=s_{1}\right) \kappa^{(n-2)}(s)$ exists and has bounded difference quotient, if, for $s=s_{1}, \kappa^{(n-1)}(s)$ and

$$
\int_{0}^{a}\left|\kappa^{(n-2)}(s+t)+\kappa^{(n-2)}(s-t)-2 \kappa^{(n-2)}(s)\right| \frac{d t}{t^{2}}
$$

exist and if $w_{1}=f(1)$, then $\lim _{r+1} f^{(n)}(r)$ exists.

(b) If $\kappa^{(n-1)}(s)$ exists over all of $c$ and is continuous, and if (5.1) exists at every point of a closed subarc $c^{\prime}$ of the open arc $c$ and approaches zero there uniformly with a, then $f^{(n)}(z)$ assumes continuous boundary values on the open arc $\gamma^{\prime}: \theta_{1}<\theta<\theta_{2}$ corresponding to $c^{\prime}$. Furthermore, if $s(\theta)$ denotes the arc length along $c$, measured from a fixed point, $s^{(n)}(\theta)$ exists on $\gamma^{\prime}$ and is continuous. $\ddagger$

(c) Suppose $R$ is the interior of a closed Jordan curve $C$ with continuous curvature of order $(n-1)$. Suppose that (5.1) exists at each point of $C$ and approaches zero with a uniformly over all of $C$. Let $\delta(\xi)$ be a modulus of continuity of $\kappa^{(n-1)}(s)$ and $\eta(\xi)$ a modulus of convergence of (5.1). Furthermore, let $D$ be a number such that the diameter of $C$ is $\leqq D$. Let $\rho>0$ be the radius of a circle with the center $f(0)=w_{0}$ lying entirely in $R$, and let $d>0$ denote a constant such that $r / \sigma \geqq d$ where $r$ is the distance between any two points of $C$ and $\sigma$ is the length of the shortest arc of $C$ joining the two points.

Then $f^{(n)}(z)$ has continuous boundary values over all of $|z|=1$ and $s^{(n)}(\theta)$ exists and is continuous there. Furthermore, there is a modulus of continuity of $f^{(n)}\left(e^{i \theta}\right)$ and $s^{(n)}(\theta), \Delta(\epsilon)$, which depends only on $\epsilon, D, \rho, d$, and on the functions $\delta(\xi)$ and $\eta(\xi)$, and there is an upper bound for $\left|f^{(n)}(z)\right|$ and $\left|s^{(n)}(\theta)\right|$ which also depends only on $D, \rho, d$, and the functions $\delta(\xi)$ and $\eta(\xi)$.

$\dagger$ That is, a Jordan arc the end points of which can be joined by another Jordan arc $b$ lying in $R$ except for the end points, such that $b$ and $c$ form a closed Jordan curve which bounds a region belonging to $R$. This idea is due to C. Carathéodory; see, for example, C. Carathéodory, Conformal Representation, Cambridge University Press, 1932, p. 86.

$\ddagger$ Theorem III (b) contains as a special case a result which can be obtained by applying to the logarithmic potential, and in particular to Green's function of a plane region, the method given by $O$. D. Kellogg in his investigations of the derivatives at the boundary, of harmonic functions in space (see footnote $\dagger \dagger$ on p. 311, second paper). The special case which is obtained in this way is the following (see Theorem III, p. 491, loc. cit.): Let $R, c$, and $w=f(z)$ have the same meanings as in Theorem III of our paper. If $\kappa^{(n-1)}(s)$ exists on $c$ and if there is a non-decreasing function $D(t)$, defined for $t>0$, for which $\int_{0}^{a}\left(D(t) / t^{2}\right) d t$ exists, such that

$$
\left|\kappa^{(n-1)}(s+t)-\kappa^{(n-1)}(s)\right| \leqq D(t), \quad t>0,
$$

then $f^{(n)}(z)$ assumes continuous boundary values on the open arc $\gamma$ corresponding to $c$.

Our proof of Theorem III (b) makes use of methods of conformal mapping and therefore admits no obvious generalization to the case of potential functions in space. 
The following theorem $†$ will be used in the proof.

ThEOREM III*. Let $R, c$, and $f(z)$ have the same meaning as in Theorem III.

(a) If $c$ has a continuously turning tangent and if the angle $\Theta(s)$, defined above, satisfies a Hölder condition on $c$ :

$$
\left|\Theta(s)-\Theta\left(s^{\prime}\right)\right| \leqq K\left|s-s^{\prime}\right|^{\beta}, 0<\beta<1,
$$

then, on any fixed closed subarc $\gamma^{\prime}$ of the open arc $\gamma$ corresponding to $c, f^{\prime}(z)$ and $s^{\prime}(\theta)=\left|f^{\prime}\left(e^{i \theta}\right)\right|$ exist, are not zero, and satisfy a Hölder condition with the same exponent:

$$
\left|s^{\prime}(\theta)-s^{\prime}\left(\theta^{\prime}\right)\right| \leqq\left|f^{\prime}\left(e^{i \theta}\right)-f^{\prime}\left(e^{i \theta^{\prime}}\right)\right| \leqq H\left|\theta-\theta^{\prime}\right|^{\beta} .
$$

(b) Suppose that $R$ is the interior of a closed Jordan curve $C$, on which (5.2) is satisfied. Then the constant $B$ in (5.3) depends only on $\beta, K$, and the three constants $D, \rho, d$ introduced in Theorem III (c). Furthermore, there are two numbers $\mu_{1}$ and $\mu_{2}$, also depending only on those five constants, such that, in $|z| \leqq 1$ : $0<\mu_{1} \leqq\left|f^{\prime}(z)\right| \leqq \mu_{2}$.

Since we shall prove Theorem III by induction, the following simple remarks will be of help to us:

If, in a neighborhood of a point $s_{1}$ of a Jordan arc $c, \kappa^{(m-1)}(s)$ exists and has bounded difference quotient, then also $\kappa^{(m-2)}(s), \kappa^{(m-3)}(s), \cdots, \kappa^{(1)}(s)$, $\Theta(s)$ have bounded difference quotients there. Hence, according to $\S 3,(1 \mathrm{a})$, each integral

$$
\int_{0}^{a}\left|\kappa^{(\nu)}(s+t)+\kappa^{(\nu)}(s-t)-2 \kappa^{(\nu)}(s)\right| \frac{d t}{t^{2}} \quad(\nu=1,2, \cdots, m-2)
$$

exists and approaches zero uniformly with $a$ in a neighborhood of $s=s_{1}$. Thus we have

Remark 1. If the hypotheses of part (a) of Theorem III are satisfied for the order $n=m+1$, then those of part (b) are satisfied for every smaller order $n=2,3, \cdots, m$, for a certain neighborhood of $s=s_{1}$. If the hypotheses of III (b) are fulfilled for the order $n=m+1$, they also are fulfilled for any smaller

$\dagger$ Part (a) of Theorem III* is a theorem of Kellogg from the first paper mentioned in footnote t† on p. 311 and is also proved in WR, p. 447. The statement of part (b) as to the dependence of $B$ on $\beta, K, D, \rho, d$ only, is proved in WR, pp. 451-452. This statement, as it is given in WR, says that $B$ depends also on a lower bound $l$ for the total length of $C$. But since we may take $l=2 \rho$, the statement about $H$ in III* (b) is true. The assertion in III* (b) concerning the existence of constants $\mu_{1}$ and $\mu_{2}$ which depend only on $\beta, K, D, \rho, d$ is covered by the theorem on p. 440 (equation (10.16)) in WR which says that $\mu_{1}$ and $\mu_{2}$ depend only on $D, \rho, d$, a lower bound $l_{1}(=2 \rho)$ for the total length of $C$, and the modulus of convergence $\delta^{\prime}(\eta)$ of the integrals (10.5) on p. 440 in WR. But since $\mid x^{\prime}(s+t)$ $-x^{\prime}(s)|=| \cos \Theta(s+t)-\cos \Theta(s)|\leqq| \Theta(s+t)-\left.\Theta(s)|\leqq K| t\right|^{\beta},\left|y^{\prime}(s+t)-y^{\prime}(s)\right| \leqq K|t|^{\beta}$, it is easily seen that $\delta^{\prime}(\eta)$ can be chosen $(\beta \eta / K)^{1 / \beta}$ and therefore depends only on $\beta$ and $K$. 
order and for any subarc of $c$ as $c^{\prime}$. In particular, we may infer from Theorem III*, using it for $\beta=\frac{1}{2}$, that under the hypotheses of part (a) or (b) or (c) of Theorem III for any $n \geqq 2$, we have relations of the form

$$
\begin{gathered}
0<\mu_{1} \leqq\left|f^{\prime}\left(e^{i \theta}\right)\right|=\left|s^{\prime}(\theta)\right| \leqq \mu_{2}, \\
\left|s^{\prime}(\theta)-s^{\prime}\left(\theta^{\prime}\right)\right| \leqq\left|f^{\prime}\left(e^{i \theta}\right)-f^{\prime}\left(e^{i \theta^{\prime}}\right)\right| \leqq H\left|\theta-\theta^{\prime}\right|^{1 / 2}
\end{gathered}
$$

holding in a neighborhood of $\theta=0$ on $|z|=1$ in case (a) $\dagger$, on any closed subarc of the open arc $\gamma$ in case (b), and over all of $|z|=1$ in case (c).

Furthermore, it follows from $\S 3,(1 \mathrm{a})$, and from (5.6) that

$$
\int_{0}^{\alpha}|s(\theta+\tau)+s(\theta-\tau)-2 s(\theta)| \frac{d \tau}{\tau^{2}} \leqq 4 H \alpha^{1 / 2} \quad(\alpha>0),
$$

if $\theta \pm \alpha$ is inside any interval for which (5.6) is true.

Another remark will be of use in proving part (c):

Suppose the hypotheses of part (c) are satisfied for $n=m+1$. Denote by $S$ the total length of $C$; evidently $2 \rho \leqq S \leqq 2 D / d$. $\ddagger$ Since

$$
\int_{0}^{S} \kappa^{(\nu)}(s) d s=\left\{\begin{array}{c}
0 \text { for } \nu>1, \\
2 \pi \text { for } \nu=1,
\end{array} \quad \nu=1,2, \cdots, m,\right.
$$

there is, according to Lemma 3 , a constant $K$, depending only on $\rho, 2 D / d$, and the modulus of continuity $\delta(\xi)$ of $\kappa^{(m)}(s)$, such that over all of $C$

$$
\left|\kappa^{(\nu)}(s)\right| \leqq K \quad(\nu=1,2, \cdots, m) .
$$

Hence, for any $s_{1}, s_{2}$,

$$
\left|\kappa^{(\nu)}\left(s_{1}\right)-\kappa^{(\nu)}\left(s_{2}\right)\right| \leqq K\left|s_{1}-s_{2}\right| \quad(\nu=1,2, \cdots, m-1),
$$

and for $0 \leqq s_{1} \leqq s_{2} \leqq S$,

$$
\left|\Theta\left(s_{1}\right)-\Theta\left(s_{2}\right)\right| \leqq K\left|s_{1}-s_{2}\right| \leqq k\left|s_{1}-s_{2}\right|^{1 / 2}, \quad k=K(2 D / d)^{1 / 2} .
$$

Thus we obtain

Remark 2. If the hypotheses of Theorem III (c) are satisfied for $n=m+1$, (5.8) holds, and every $\kappa^{(\nu)}(s), \nu=1,2, \cdots, m-1$, has a modulus of continuity which depends only on $\epsilon, D, \rho, d$, and on the modulus of continuity $\delta(\xi)$ of $\kappa^{(m)}(s)$. Furthermore, because of (5.9), each integral (5.4) which approaches 0 with $a$ uniformly on $C$, has a modulus of convergence also depending only on $\epsilon, D, \rho, d$, and on the function $\delta(\xi)$. Because of (5.10) it follows from Theorem

$\dagger$ It should be noticed that (5.5) is true on a closed neighborhood of $\theta=0$ under hypothesis (a) of Theorem III* because $f^{\prime}(z)$ is continuous and different from 0 there.

$\ddagger$ In order to prove $S \leqq 2 D / d$ we need only apply the inequality $r / \sigma \geqq d$ to two points on $C$ dis$\operatorname{tant} \sigma=\frac{1}{2} S$ from each other along $C$. 
III* (b) that $\mu_{1}, \mu_{2}$ in (5.5) and $H$ in (5.6) and (5.7) depend only on $D, \rho, d$, and on $\delta(\xi)$.

6. Proof of Theorem III. We shall first show that if the theorem is true for the orders $n=2,3, \cdots, m$, it is also true for $n=m+1$. In the proof of each of the three parts we thus assume that all three parts have been proved for $n=2,3, \cdots, m$ and the hypotheses of the part in question are satisfied for $n=m+1$.

(1) Since $f^{\prime}(z)$ is not zero in $|z|<1$ every branch of $\log f^{\prime}(z)$ is regular there. We choose a branch of $\log f^{\prime}(z)$ and note first that our proof will be completed if we prove, in each of the three cases, that the function $\partial^{m} \log f^{\prime}(z) / \partial \theta^{m}, z=r e^{i \theta}$, satisfies the result stated in the conclusion about $f^{(m+1)}(z)$. For we have, first,

$$
\frac{\partial^{m} \log f^{\prime}(z)}{\partial \theta^{m}}=\frac{d^{m} \log f^{\prime}(z)}{d z^{m}}\left(\frac{\partial z}{\partial \theta}\right)^{m}+P_{m}(z),
$$

where $P_{m}(z)$ is a polynomial in the derivatives of $\log f^{\prime}(z)$ with respect to $z$ of order $\leqq m-1$ and in derivatives of $z$ with respect to $\theta$. By replacing each expression $d^{\mu} \log f^{\prime}(z) / d z^{\mu}$ by

$$
\frac{d^{\mu-1}}{d z^{\mu-1}}\left[\frac{f^{\prime \prime}(z)}{f^{\prime}(z)}\right]=\sum_{\beta=0}^{\mu-1}\left(\begin{array}{c}
\mu-1 \\
\beta
\end{array}\right) \frac{d^{\beta} f^{\prime \prime}(z)}{d z^{\beta}} \frac{d^{\mu-\beta-1}}{d z^{\mu-\beta-1}}\left[\frac{1}{f^{\prime}(z)}\right],
$$

we obtain, by use of $\partial^{\mu} z / \partial \theta^{\mu}=i^{\mu} z$,

$$
\frac{\partial^{m} \log f^{\prime}(z)}{\partial \theta^{m}}=(i z)^{m} \frac{f^{(m+1)}(z)}{f^{\prime}(z)}+R_{m}(z),
$$

where $R_{m}(z)$ is a polynomial in the derivatives of $f(z)$ of order $\leqq m$, in $z$ and $1 / f^{\prime}(z)$.

We supposed that the hypotheses of part (a), (b), or (c) as the case may be are satisfied for $n=m+1$. According to Remarks 1 and 2 above, we may therefore in each of the three cases apply the corresponding part of our theorem about $f^{\prime \prime}(z), f^{\prime \prime \prime}(z), \cdots, f^{(m)}(z)$, which is supposed to be proved, and also the results about $f^{\prime}(z)$ stated in these remarks. Since this shows that $R_{m}(z)$ has the properties which are to be established for $f^{(m+1)}(z)$, it follows from (6.1) that $f^{(m+1)}(z)$ will have these properties, if $\partial^{m} \log f^{\prime}(z) / \partial \theta^{m}$ has them.

In order to infer also the result of part (b) or (c) about $s^{(m+1)}(\theta)$ from this property of $\partial^{m} \log f^{\prime}(z) / \partial \theta^{m}$, we first observe that, if $\partial^{m} \log f^{\prime}\left(r e^{i \theta}\right) / \partial \theta^{m}$ $(0<r<1)$ has continuous boundary values on an arc $\theta_{1}<\theta<\theta_{2}$ of $|z|=1$, also $d^{m} \log f^{\prime}\left(e^{i \theta}\right) / d \theta^{m}$ exists on this arc and is equal to $\lim _{r+1} \partial^{m} \log f^{\prime}\left(r e^{i \theta}\right) / \partial \theta^{m}$. Therefore, it follows from 


$$
\begin{aligned}
\frac{d^{m} \log \left|f^{\prime}\left(e^{i \theta}\right)\right|}{d \theta^{m}} & =\frac{d^{m} \log s^{\prime}(\theta)}{d \theta^{m}}=\frac{d}{d \theta}\left[\frac{d^{m-2}}{d \theta^{m-2}}\left(\frac{s^{\prime \prime}(\theta)}{s^{\prime}(\theta)}\right)\right] \\
& =\frac{d}{d \theta}\left[s^{(m)}(\theta) \frac{1}{s^{\prime}(\theta)}+\cdots+s^{\prime \prime}(\theta) \frac{d^{m-2}}{d \theta^{m-2}} \frac{1}{s^{\prime}(\theta)}\right]
\end{aligned}
$$

that $s^{(m+1)}(\theta)$ exists on $\gamma^{\prime}$ or over all of $|z|=1$ respectively, since $s^{\prime}(\theta) \neq 0$ there. Hence, we have

$$
\frac{d^{m} \log s^{\prime}(\theta)}{d \theta^{m}}=s^{(m+1)}(\theta) \frac{1}{s^{\prime}(\theta)}+\cdots+s^{\prime \prime}(\theta) \frac{d^{m-1}}{d \theta^{m-1}} \frac{1}{s^{\prime}(\theta)},
$$

from which we easily infer the result about the continuity of $s^{(m+1)}(\theta)$ in case (b) and the modulus of continuity and the bound of $s^{(m+1)}(\theta)$ in case (c).

(2) We now prove the result concerning $\partial^{m} \log f^{\prime}(z) / \partial \theta^{m}$. Let us first assume that the hypotheses of part (a) are satisfied for $n=m+1$. According to Remark 1 above, the hypotheses of part (b) of our theorem are then also satisfied in a neighborhood of $s=s_{1}$ for $n=2,3, \cdots, m$. Hence, if we set $\Theta^{*}(\theta)=\Theta(s(\theta))$, we see that

$$
V(\theta) \equiv \frac{d^{m-1}}{d \theta^{m-1}} \Theta^{*}(\theta)=\kappa^{(m-1)}(s)\left(s^{\prime}(\theta)\right)^{m-1}+\cdots+\kappa^{(1)}(s) s^{(m-1)}(\theta)
$$

exists in a neighborhood of $\theta=0$. Furthermore, since $\kappa^{(m)}(s)$ exists for $s=s_{1}$ and $s^{(m)}(\theta)$ at $\theta=0$, also

$$
V^{\prime}(\theta)=\frac{d^{m}}{d \theta^{m}} \Theta^{*}(\theta)=\kappa^{(m)}(s)\left(s^{\prime}(\theta)\right)^{m}+\cdots+\kappa^{(1)}(s) s^{(m)}(\theta)
$$

exists at $\theta=0$.

The functions $\kappa^{(\nu)}(s)$ and $s^{(\nu)}(\theta), \nu=1,2, \cdots, m-1$, have bounded difference quotients in a neighborhood of $s=s_{1}$ and $\theta=0$ respectively. Hence $V(\theta)$ has this property in an interval $-\delta \leqq \theta \leqq \delta(0<\delta \leqq \pi)$ :

$$
\left|V\left(\theta_{1}\right)-V\left(\theta_{2}\right)\right| \leqq k\left|\theta_{1}-\theta_{2}\right| \leqq k(2 \pi)^{1 / 2}\left|\theta_{1}-\theta_{2}\right|^{1 / 2} \text {. }
$$

By using (6.1) with $m$ replaced by $m-1$, we see that $\partial^{m-1} \log f^{\prime}(z) / \partial \theta^{m-1}$ assumes continuous boundary values on $|z|=1$ in a neighborhood of $z=1$. Since there, for a suitable branch of arc $f^{\prime}(z)$, arc $f^{\prime}\left(e^{i \theta}\right)=\Theta^{*}(\theta)-\theta-\pi / 2$, it is easily seen that the boundary function of the imaginary part of $\partial^{m-1} \log f^{\prime}(z) / \partial \theta^{m-1}$ is $V(\theta)$, if $m>2$, and $V(\theta)-1$, if $m=2$. According to a theorem of Privaloff, $\dagger$ it follows from the fact that $V(\theta)$ satisfies a Hölder condition with the exponent $\frac{1}{2}$ for $-\delta \leqq \theta \leqq \delta$, that also the real part of the boundary function of $\partial^{m-1} \log f^{\prime}(z) / \partial \theta^{m-1}$, that is, $d^{m-1} \log s^{\prime}(\theta) / d \theta^{m-1}$, satis-

† I. Privaloff, Bulletin de la Société Mathématique de France, vol. 44 (1916), pp. 100-103. 
fies a Hölder condition with the same exponent in any fixed interval $-\delta^{\prime} \leqq \theta \leqq \delta^{\prime}$ with $0<\delta^{\prime}<\delta$ :

$$
\left|\left[\frac{d^{m-1} \log s^{\prime}(\theta)}{d \theta^{m-1}}\right]_{\theta=\theta_{1}}-\left[\frac{d^{m-1} \log s^{\prime}(\theta)}{d \theta^{m-1}}\right]_{\theta=\theta_{2}}\right| \leqq h\left|\theta_{1}-\theta_{2}\right|^{1 / 2} .
$$

For the proof of part (c) of our theorem it is important to notice that, if $V(\theta)$ satisfies the Hölder condition in (6.4) over all of $|z|=1$, the proof of Privaloff's theorem implies that also (6.5) is true over all of $|z|=1$ and that the constant $h$ in (6.5) depends only on the constant $k$ in (6.4).

From (6.5) we infer with the help of (6.2), used for $m-1$ instead of $m$, that, in the neighborhood of $\theta=0$, also

$$
\left.\left|s^{(m)}\left(\theta_{1}\right)-s^{(m)}\left(\theta_{2}\right)\right| \leqq g\left|\theta_{1}-\theta_{2}\right|^{1 / 2} \quad \text { (g constant }\right) .
$$

Now we note that we may write (6.3) in the form

$$
V(\theta)=\kappa^{(m-1)}(s)\left(s^{\prime}(\theta)\right)^{m-1}+\Pi_{m}(\theta)
$$

where $\Pi_{m}(\theta)$ denotes a polynomial in $\kappa^{(\nu)}(s(\theta)), \nu=1,2, \cdots, m-2$, and $s^{(\nu)}(\theta)$, $\nu=1,2, \cdots, m-1$. Since by Remark 1 each integral (5.4) exists at the point $s=s_{1}$, it follows from $\S 3,(3)$, and (5.5) and (5.7) that also the integrals

$$
\int_{0}^{\alpha}\left|\kappa^{(\nu)}(s(\theta+\tau))+\kappa^{(\nu)}(s(\theta-\tau))-2 \kappa^{(\nu)}(s)\right| \frac{d \tau}{\tau^{2}} \quad(\nu=1,2, \cdots, m-2)
$$

exist at $\theta=0$ for an $\alpha>0$. Furthermore, since in a neighborhood of $\theta=0$ $s^{(\nu)}(\theta), \nu=2,3, \cdots, m-1$, have bounded difference quotients and $s^{(m)}(\theta)$ satisfies the Hölder condition (6.6), it follows from $\$ 3,(1 \mathrm{a}),(3.3)$, that the integrals

$$
\int_{0}^{\alpha}\left|s^{(\nu)}(\theta+\tau)+s^{(\nu)}(\theta-\tau)-2 s^{(\nu)}(\theta)\right| \frac{d \tau}{\tau^{2}} \quad(\nu=1,2, \cdots, m-1)
$$

exist at $\theta=0$ for an $\alpha>0$. According to $\S 3,(2)$, therefore

$$
\int_{0}^{\alpha}\left|\Pi_{m}(\theta+\tau)+\Pi_{m}(\theta-\tau)-2 \Pi_{m}(\theta)\right| \frac{d \tau}{\tau^{2}}
$$

exists at $\theta=0$.

Now we recall that by hypothesis the integral

$$
\int_{0}^{a}\left|\kappa^{(m-1)}(s+t)+\kappa^{(m-1)}(s-t)-2 \kappa^{(m-1)}(s)\right| \frac{d t}{t^{2}}
$$

exists at $s=s_{1}$. Because of (5.5) and (5.7) we may apply $\S 3,(3)$, once more and we see that also 


$$
\int_{0}^{\alpha}\left|\kappa^{(m-1)}(s(\theta+\tau))+\kappa^{(m-1)}(s(\theta-\tau))-2 \kappa^{(m-1)}(s(\theta))\right| \frac{d \tau}{\tau^{2}}
$$

exists at $\theta=0$ for an $\alpha>0$. Set $\Omega_{m}(\theta)=\kappa^{(m-1)}(s(\theta))\left(s^{\prime}(\theta)\right)^{m-1}$. According to $\S 3$, (2), (3.5), therefore

$$
\int_{0}^{\alpha}\left|\Omega_{m}(\theta+\tau)+\Omega_{m}(\theta-\tau)-2 \Omega_{m}(\theta)\right| \frac{d \tau}{\tau^{2}}
$$

exists at $\theta=0$ for an $\alpha>0$. From (6.7) and (6.9) we finally infer, with the help of (3.4), that

$$
\int_{0}^{\alpha}|V(\theta+\tau)+V(\theta-\tau)-2 V(\theta)| \frac{d \tau}{\tau^{2}}
$$

exists at $\theta=0$.

Let us now make a few remarks concerning the proof of part (b) and (c) of our theorem. If the hypotheses of (b) and (c) are satisfied, we see by examining part (2) of our proof above once more and by using Remarks 1 and 2:

First, that $V^{\prime}(\theta)$ is continuous on the open arc $\gamma$ or over all of $|z|=1$ respectively, and that in the latter case the modulus of continuity, $\delta^{*}(\epsilon)$, of $V^{\prime}(\theta)$ depends only on $\epsilon, D, \rho, d$, and the modulus of continuity, $\delta(\xi)$, of $\kappa^{(m)}(s)$. Furthermore, in this case $k$ in (6.4), hence $g$ in (6.6), also depend only on $D, \rho, d$ and $\delta(\xi)$.

Secondly, we see that the integral (6.7) approaches zero uniformly with $\alpha$ on every closed subarc of the open arc $\gamma$ or over all of $|z|=1$ respectively, and that in the latter case the modulus of convergence of (6.7) depends only on the same things as $\delta^{*}(\epsilon)$.

Thirdly, we see that (6.9) also, hence (6.10), approach zero uniformly with $\alpha$ on the arc $\gamma^{\prime}$ or over all of $|z|=1$ respectively, and that in case (c) the modulus of convergence $\eta^{*}(\epsilon)$ of (6.10) depends only on $\epsilon, D, \rho, d$, and the modulus of continuity $\delta(\xi)$ of $\kappa^{(m)}(s)$, and the modulus of convergence $\eta(\xi)$ of (6.8).

Now we can complete the proof of all three parts simultaneously.

The function $V(\theta)-d^{m-1} \theta / d \theta^{m-1}$ is the boundary function of the imaginary part of

$$
F(z) \equiv \frac{\partial^{m-1} \log f^{\prime}(z)}{\partial \theta^{m-1}}, z=r e^{i \theta}
$$

in a neighborhood of $\theta=0$ on $|z|=1$ in case (a), on the open arc $\gamma$ in case (b), and on $|z|=1$ in case (c). Since, under the hypotheses of part (a), $V^{\prime}(\theta)$ and (6.10) exist at $\theta=0$, the result which we wish to prove follows immediately from Lemma 5 (a). According to the three remarks which we have just made 
concerning parts (b) and (c), these parts follow from Lemmas 5 (b) and 4 (c) respectively.

To complete the induction we have to show now that Theorem III is true for $n=2$. We obtain this proof for $n=2$ immediately from the preceding proof by setting $m+1=2$, since all properties of $f^{\prime}(z)$ which are used in this proof are based on Theorem III*. This completes the proof of Theorem III.

7. Existence of the $n$th derivative of the mapping function at a boundary point. We prove the following theorem:

TheOREM IV. Let $R$ be a simply connected region. Let the boundary of $R$ contain a free Jordan arc c. Suppose that the arc $c$ has an $L$-curvature of order $n-1$ at an interior point $w_{1}\left(s=s_{1}\right)$ and that

$$
\int_{0}^{a}\left|\kappa^{(n-2)}\left(s_{1}+t\right)+\kappa^{(n-2)}\left(s_{1}-t\right)-2 \kappa^{(n-2)}\left(s_{1}\right)\right| \frac{d t}{t^{2}}
$$

exists for an $a>0$. If $w=f(z)$ is a function which maps the circle $|z|<1$ on $R$ in such a manner that $z=1$ corresponds to $w_{1}$, then $f^{(n-1)}(z)$ assumes continuous boundary values on $|z|=1$ in a neighborhood of $z=1$ and is differentiable at $z=1$.

We shall use one part of the following lemma in the proof.

LEMMA 6. Let $v(z)$ be harmonic in the circle $|z|<1$ and assume continuous boundary values $V(\theta)$ on $|z|=1$. A necessary and sufficient condition that

$$
\lim _{z \rightarrow 1} \frac{\partial v\left(r e^{i \phi}\right)}{\partial \phi}, z=r e^{i \phi}
$$

exist for unrestricted approach in $|z|<1$ is that

$$
\lim \frac{V(\phi)-V\left(\phi^{\prime}\right)}{\phi-\phi^{\prime}}=V^{\prime}(0)
$$

exist when $\phi$ and $\phi^{\prime}$ approach zero simultaneously. The limits $\lim _{z \rightarrow 1} \partial v\left(r e^{i \phi}\right) / \partial \phi$ and (7.2) are equal if one of them exists.

Proof of Lemma 6. (1) If (7.2) exists, then, for almost every $\alpha$ of a suitable interval $-\delta \leqq \alpha \leqq \delta, V^{\prime}(\alpha)$ exists and is continuous at $\alpha=0$. Furthermore, for every $\alpha$ of this interval, $V(\alpha)-V(0)=\int_{0}^{\alpha} V^{\prime}(\phi) d \phi$, the integral being taken in the sense of Lebesgue. From Poisson's formula

$$
v\left(r e^{i \phi}\right)=\frac{1}{2 \pi} \int_{-\pi}^{\pi} V(\alpha) K(r, \alpha-\phi) d \alpha, \quad K(r, \psi) \equiv \frac{1-r^{2}}{1+r^{2}-2 r \cos \psi},
$$

which holds for $r<1$, we obtain 


$$
\begin{aligned}
\frac{\partial v\left(r e^{i \phi}\right)}{\partial \phi} & =\frac{1}{2 \pi} \int_{-\pi}^{\pi} V(\alpha) \frac{\partial K(r, \alpha-\phi)}{\partial \phi} d \alpha=-\frac{1}{2 \pi} \int_{-\pi}^{\pi} V(\alpha) \frac{\partial K(r, \alpha-\phi)}{\partial \alpha} d \alpha \\
& =-\frac{1}{2 \pi}\left\{\int_{-\delta}^{\delta}+\int_{\delta}^{\pi}+\int_{-\pi}^{-\delta}\right\} .
\end{aligned}
$$

By integrating the integral $\int_{-\delta}^{\delta} V(\alpha)(\partial K / \partial \alpha) d \alpha$ by parts, we thus get

$$
\begin{aligned}
\frac{\partial v\left(r e^{i \phi}\right)}{\partial \phi}= & \frac{1}{2 \pi} \int_{-\delta}^{\delta} V^{\prime}(\alpha) K(r, \alpha-\phi) d \alpha+V(-\delta) K(r, \delta+\phi)-V(\delta) K(r, \delta-\phi) \\
& -\frac{1}{2 \pi}\left\{\int_{\delta}^{\pi}+\int_{-\pi}^{-\delta}\right\} .
\end{aligned}
$$

Since $\lim _{\phi \rightarrow 0} V^{\prime}(\phi)=V^{\prime}(0)$ when $\phi \rightarrow 0$ over the point set on which $V^{\prime}(\phi)$ is defined, it follows by a well known procedure that $\lim _{z \rightarrow 1} \partial v\left(r e^{i \phi}\right) / \partial \phi$ exists for unrestricted approach in $|z|<1$ and $=V^{\prime}(0)$.

(2) If, conversely, $\lim _{z \rightarrow 1} \partial v\left(r e^{i \phi}\right) / \partial \phi, z=r e^{i \phi}$, exists, then there is a $\delta>0$ such that $\partial v / \partial \phi$ is bounded in the sector $\{-\delta \leqq \phi \leqq \delta, 0<r<1\}$. According to Fatou's theorem, $\partial v\left(r e^{i \phi}\right) / \partial \phi$ therefore has radial boundary values $h(\phi)$ for almost every $\phi$ in $-\delta \leqq \phi \leqq \delta$. Evidently $h(\phi)$ is continuous at $\phi=0$. Hence, according to Lebesgue's integral theorem, we have

$$
V(\phi)-V(0)=\lim _{r \uparrow 1} \int_{0}^{\phi} \frac{\partial v\left(r e^{i \alpha}\right)}{\partial \alpha} d \alpha=\int_{0}^{\phi} \lim _{r \uparrow 1} \frac{\partial v\left(r e^{i \alpha}\right)}{\partial \alpha} d \alpha=\int_{0}^{\phi} h(\alpha) d \alpha,
$$

from which (7.2) with $V^{\prime}(0)=h(0)$ is easily inferred.

Proof of Theorem IV. According to the Theorems III* and III (b), $f^{\prime}(z)$, $f^{\prime \prime}(z), \cdots, f^{(n-1)}(z)$ assume continuous boundary values on a certain $\operatorname{arc} \gamma^{*}$ : $-\delta \leqq \theta \leqq \delta$ of $|z|=1$, and according to III (a) $\lim _{r+1} f^{(n)}(r)$ exists. If we denote, as before, by $s(\theta)$ the arc length along $c$, then also $s^{\prime}(\theta), \cdots, s^{(n-1)}(\theta)$ are defined and continuous in $-\delta \leqq \theta \leqq \delta$.

We choose a fixed branch of $\log f^{\prime}(z)$ and consider $G^{*}(z) \equiv \partial^{n-2} \log f^{\prime}(z)$ $/ \partial \theta^{n-2},|z|=\left|r e^{i \theta}\right|<1$. With the help of (6.1), used for $m=n-2$, it follows from what we have just noted about $f^{\prime}(z), \cdots, f^{(n-1)}(z)$, and $f^{(n)}(z)$ that $G^{*}(z)$ assumes continuous boundary values on $\gamma^{*}$ and that $\lim _{r+1} G^{* \prime}(r)$ exists.

Let $\Theta(s)$ denote the angle defined as in the beginning of $\S 5$. If $\Theta^{*}(\theta)$ $=\Theta(s(\theta))$, then $\Im\left(\log f^{\prime}\left(e^{i \theta}\right)\right)=\Theta^{*}(\theta)-\theta+C$ on $\gamma^{*}, C$ being a constant which depends on the branch of $\log f^{\prime}(z)$ chosen by us, and therefore

$$
\begin{aligned}
V^{*}(\theta) \equiv & \frac{d^{n-2}}{d \theta^{n-2}}\left[\Theta^{*}(\theta)-\theta+C\right]=\kappa^{(n-2)}(s)\left(s^{\prime}(\theta)\right)^{n-2}+\cdots+\kappa^{(1)}(s) s^{(n-2)}(\theta) \\
& -\frac{d^{n-2}}{d \theta^{n-2}}[\theta-C]
\end{aligned}
$$


is the boundary function of the imaginary part of $G^{*}(z)$. Furthermore, it follows from our hypothesis that

$$
\lim \frac{V^{*}(\theta)-V^{*}\left(\theta^{\prime}\right)}{\theta-\theta^{\prime}}=V^{* \prime}(0)
$$

exists when $\theta$ and $\theta^{\prime}$ approach zero simultaneously.

Let $\Gamma$ denote the closed Jordan curve formed by $\gamma^{*}$ and the part within $|z|<1$ of the circle with center $z=1$ through $z=e^{i \delta}\left(\right.$ and $\left.e^{-i \delta}\right) . \Gamma$ is symmetric in the axis of reals. Let $z=g(\zeta)$ be the function which maps the circle $|\zeta|<1$ on the interior of $\Gamma$. in such a manner that $g(1)=1$ and that the segment $-1<\zeta<1$ corresponds to the part of the real axis which lies in the interior of $\Gamma$. The function $z=g(\zeta)$ is also analytic on the open $\operatorname{arc} \gamma$ of $|\zeta|=1$ which corresponds to $\gamma^{*}$, and $g^{\prime}(\zeta) \neq 0$ here. $G(\zeta)=G^{*}(g(\zeta))$ is regular in $|\zeta|<1$ and assumes continuous boundary values on $|\zeta|=1$. The boundary function of its imaginary part on $\gamma$ is $V(\phi)=V^{*}\left(\operatorname{arc} g\left(e^{i \phi}\right)\right)$. Evidently

$$
\lim \left(V(\phi)-V\left(\phi^{\prime}\right)\right) /\left(\phi-\phi^{\prime}\right)
$$

exists as $\phi$ and $\phi^{\prime}$ approach zero simultaneously. Hence, according to Lemma 6,

$$
\lim _{\zeta \rightarrow 1} \Im\left(\frac{\partial G(\zeta)}{\partial \phi}\right), \quad \zeta=\rho e^{i \phi},
$$

exists for unrestricted approach in $|\zeta|<1$. Furthermore,

$$
\lim _{\rho \uparrow 1} G^{\prime}(\rho)=\lim _{r \uparrow 1} G^{* \prime}(r) \cdot \lim _{\rho \uparrow 1} g^{\prime}(\rho)
$$

exists. Finally, we have, for $0<\rho<1$ :

$$
\left[\frac{\partial G(\zeta)}{\partial \phi}\right]_{\zeta=0}=\frac{1}{2 \pi} \int_{0}^{2 \pi} \frac{\partial G\left(\rho e^{i \phi}\right)}{\partial \phi} d \phi=0 .
$$

Therefore we may apply Theorem II with $h(\zeta)=\partial G(\zeta) / \partial \phi$, according to which

$$
G(\zeta)-G(0)=\int_{0}^{\zeta} G^{\prime}(u) d u=\frac{1}{i} \int_{0}^{\zeta} \frac{h(u)}{u} d u
$$

has a derivative at $\zeta=1$. Hence $G^{*}(z)$ has a derivative at $z=1$. With the help of (6.1), used for $m=n-2$, we easily infer from this that $f^{(n-1)}(z)$ is also differentiable at $z=1$. 


\section{A CONVERGENCE THEOREM}

8. Statement of Theorem $\mathrm{V}$ and a preliminary remark. The theorem in question is as follows:

Theorem V. Let $C_{m}(m=1,2, \cdots)$ and $C$ be closed Jordan curves, which are represented by the following functions, differentiable for $0 \leqq t \leqq T$ :

$$
\begin{aligned}
& w=W_{m}(t)=U_{m}(t)+i V_{m}(t), W_{m}^{\prime}(t) \neq 0 \quad(m=1,2, \cdots) ; \\
& w=W(t), W^{\prime}(t) \neq 0 . \dagger
\end{aligned}
$$

Suppose

(1) that $W_{m}(t) \rightarrow W(t)$ for each $t$ in $0 \leqq t \leqq T$ as $m \rightarrow \infty$,

(2) that $W_{m}(t)$ has a continuous nth derivative $W_{m}^{(n)}(t), n \geqq 1$, which converges uniformly in $0 \leqq t \leqq T$ as $m \rightarrow \infty$,

(3) that the integrals

$$
\begin{aligned}
& \int_{0}^{a}\left|W_{m}^{\prime}(t \pm u)-W_{m}^{\prime}(t)\right| \frac{d u}{u} \quad \text { if } n=1, \\
& \int_{0}^{a}\left|W_{m}^{(n-1)}(t+u)+W_{m}^{(n-1)}(t-u)-2 W_{m}^{(n-1)}(t)\right| \frac{d u}{u^{2}} \quad \text { if } n>1,
\end{aligned}
$$

exist and approach zero with a uniformly for all $t$ in $0 \leqq t \leqq T$ and all $m=1$, $2, \cdots . \ddagger$

Suppose, further, that there is a point $w_{0}$ in the interior $R_{m}$ of $C_{m}$ for every $m$ and in the interior $R$ of $C$. Let $f_{m}(z)$ and $f(z)$ map the circle $|z|<1$ on $R_{m}$ or $R$ respectively in such a manner that $f_{m}(0)=f(0)=w_{0}$ and $f_{m}^{\prime}(0)>0, f^{\prime}(0)>0$. Then the functions $f_{m}^{(n)}(z)$ and $f^{(n)}(z)$ assume continuous boundary values on $|z|=1$, and $f_{m}^{(n)}(z)$ converges uniformly in $|z| \leqq 1$ toward $f^{(n)}(z)$ as $m \rightarrow \infty$.

Before we prove this theorem we shall discuss a few simple consequences of the hypotheses (1) and (2) in the following

Remark. As is well known, hypothesis (2) implies that the family of functions $W_{m}^{(n)}(t), m=1,2, \cdots$, is equicontinuous $\S$ for $0 \leqq t \leqq T$ and all

$\dagger$ Of course, $W_{m}(t)$ and $W(t)$ are defined for all real $t$, by the equations $W_{m}(t+T)=W_{m}(t)$, $W(t+T)=W(t)$.

$\ddagger$ The difference in the nature of the two hypotheses (for $n=1$ and for $n>1$ ) in (3) is due to the difference between the types of conditions which we have obtained for existence and continuity of the derivatives at the boundary in these cases.

As a corollary, Theorem $\mathrm{V}$ is true if hypotheses (2) and (3) are replaced by the condition that there be a non-decreasing function $D(t)$ for which $\int_{0}^{a}(D(t) / t) d t$ converges, such that, for all $t$ and $m$, $\left|W_{m}^{(n)}(t+u)-W_{m}{ }^{(n)}(t)\right| \leqq D(u), u>0$.

$\S$ A family of functions $\phi_{n}(x), n=1,2, \cdots$, defined for $a \leqq x \leqq b$, is said to be equicontinuous, if for every $\epsilon>0$ there is a $\delta(\epsilon)>0$ such that $\left|\phi_{n}(x)-\phi_{n}\left(x^{\prime}\right)\right| \leqq \epsilon$ if $\left|x-x^{\prime}\right| \leqq \delta(\epsilon)$, uniformly for all $x$, $x^{\prime}$ in $a \leqq x \leqq b$ and all $n=1,2, \cdots$. 
$m=1,2, \cdots$. Since $\int_{0}^{T} W_{m}{ }^{(v)}(t) d t=0, \nu=1,2, \cdots, n$, it follows from Lemma 3 that there is a constant $k_{1}$ such that for $0 \leqq t \leqq T$ and $m=1,2, \cdots$,

$$
\left|W_{m}^{(\nu)}(t)\right| \leqq k_{1} \quad(\nu=1,2, \cdots, n) .
$$

With the help of (8.1) and the hypotheses (1) and (2) we shall prove that also $W(t)$ has a continuous nth derivative $W^{(n)}(t)$ and that $W_{m}^{(\nu)}(t)$ approaches $W^{(v)}(t)(\nu=0,1,2, \cdots, n)$ uniformly for $0 \leqq t \leqq T$ and thus for all real $t$.

First we note that because of (8.1) we can choose from each subsequence of $\left[W_{m}(t)\right]$ a subsequence $\left[W_{m_{k}}(t)\right]$ such that $\lim _{k \rightarrow \infty} W_{m_{k}}{ }^{(\nu)}(0)=c_{\nu} \quad(\nu=1$, $2, \cdots, n-1)$ exists. Let $\Phi^{(n)}(t)$ denote the (continuous) function toward which $W_{m}^{(n)}(t)$ converges. Set

$$
\Phi^{(\nu-1)}(t)=\int_{0}^{t} \Phi^{(\nu)}(\tau) d \tau+c_{\nu} \quad(\nu=1,2, \cdots, n) .
$$

Then it follows from

$$
W_{m}^{(\nu-1)}(t)=\int_{0}^{t} W_{m}^{(\nu)}(\tau) d \tau+W_{m}^{(\nu-1)}(0)
$$

that $W_{m_{k}}^{(\nu)}(t)$ approaches $\Phi^{(\nu)}(t)$ uniformly $(\nu=0,1,2, \cdots, n-1)$ in $0 \leqq t \leqq T$ when $k \rightarrow \infty$. Since by hypothesis (1) $\lim _{m \rightarrow \infty} W_{m}(t)=W(t)$, we have $\Phi^{(0)}(t) \equiv W(t)$. Therefore $W(t)$ has a continuous $n$th derivative and $W_{m_{k}}{ }^{(\nu)}(t)$ approaches $W^{(v)}(t)$ uniformly $(\nu=1,2, \cdots, n)$. Since every subsequence of $\left[W_{m}(t)\right]$ contains a subsequence $\left[W_{m_{k}}(t)\right]$, for which $W_{m_{k}}{ }^{(\nu)}(t)$ always approaches uniformly the same limit function $W^{(v)}(t)$, it follows that the sequences $W_{m}^{(\nu)}(t)$ themselves approach $W^{(\nu)}(t)$ uniformly, $\nu=0,1, \cdots, n$.

We shall need the following two corollaries of the fact that $W_{m}^{\prime}(t)$ approaches $W^{\prime}(t)$ uniformly.

a. Since $W_{m}^{\prime}(t) \neq 0$ and $W^{\prime}(t) \neq 0$, there is evidently a constant $k_{0}$ such that

$$
\left|W_{m}^{\prime}(t)\right| \geqq k_{0} \quad(m=1,2, \cdots), \quad\left|W^{\prime}(t)\right| \geqq k_{0} \quad(0 \leqq t \leqq T) .
$$

b. There is a constant $d>0$, independent of $m$, such that

$$
\frac{r}{\sigma} \geqq d>0
$$

where $r$ is the distance between any two points $P_{1}$ and $P_{2}$ on $C_{m}(m=1,2, \cdots)$ and $\sigma$ is the length of the shortest arc of $C_{m}$ joining the two points.

To prove (8.3), we note first that we can always assign to $P_{1}$ and $P_{2}$ values $t_{1}$ and $t_{2}$ of the parameter $t$ such that $\left|t_{1}-t_{2}\right| \leqq T / 2$ and $0 \leqq t_{1}, t_{2} \leqq 2 T$. According to the mean-value theorem, we have 


$$
\frac{r^{2}}{\sigma^{2}} \geqq \frac{\left|W_{m}\left(t_{1}\right)-W_{m}\left(t_{2}\right)\right|^{2}}{\left(\int_{t_{2}}^{t_{1}}\left|W_{m}^{\prime}(t)\right| d t\right)^{2}}=\frac{U_{m}^{\prime 2}\left(\tau_{1}\right)+V_{m}^{\prime 2}\left(\tau_{2}\right)}{\left|W_{m}^{\prime}\left(\tau_{3}\right)\right|^{2}}
$$

where $\tau_{1}, \tau_{2}, \tau_{3}$ are numbers between $t_{1}$ and $t_{2}$. Since the sequence $W_{m}^{\prime}(t)$ is equicontinuous for $0 \leqq t \leqq 2 T, m=1,2, \cdots$, there is a $\delta>0$ such that

$$
\left|W_{m}^{\prime}\left(t^{\prime}\right)-W_{m}^{\prime}\left(t^{\prime \prime}\right)\right| \leqq \frac{k_{0}^{2}}{8 k_{1}} \text { for }\left|t^{\prime}-t^{\prime \prime}\right| \leqq \delta, \quad 0 \leqq t^{\prime}, t^{\prime \prime} \leqq 2 T,
$$

where $k_{0}$ and $k_{1}$ denote the constants in (8.1) and (8.2). Hence, if $\left|t_{1}-t_{2}\right| \leqq \delta$,

$$
\begin{aligned}
\frac{r^{2}}{\sigma^{2}} & \geqq \frac{\left|W_{m}^{\prime}\left(\tau_{3}\right)\right|^{2}-\left|U_{m}^{\prime 2}\left(\tau_{3}\right)-U_{m}^{\prime 2}\left(\tau_{1}\right)\right|-\left|V_{m}^{\prime 2}\left(\tau_{3}\right)-V_{m}^{\prime 2}\left(\tau_{2}\right)\right|}{\left|W_{m}^{\prime}\left(\tau_{3}\right)\right|^{2}} \\
& \geqq 1-2 \frac{2 k_{1}}{k_{0}^{2}} \frac{k_{0}^{2}}{8 k_{1}}=\frac{1}{2} .
\end{aligned}
$$

If $\left|t_{1}-t_{2}\right| \leqq \delta$ this proves (8.3). Let $P_{1}$ and $P_{2}$ be points for which $\left|t_{1}-t_{2}\right|>\delta$. As $\left|W_{m}\left(t_{1}\right)-W_{m}\left(t_{2}\right)\right|-\left|W\left(t_{1}\right)-W\left(t_{2}\right)\right|$ and

$$
\int_{t_{1}}^{t_{2}}\left[\left|W_{m}^{\prime}(t)\right|-\left|W^{\prime}(t)\right|\right] d t
$$

approach zero uniformly for $0 \leqq t_{1}, t_{2} \leqq 2 T$ when $m \rightarrow \infty$ and

$$
\left|\int_{t_{1}}^{t_{2}}\right| W_{m}^{\prime}(t)|d t| \geqq k_{0} \delta,
$$

(8.3) holds for all $C_{m}$ for which $m$ is sufficiently great, since (8.3) is valid if $P_{1}$ and $P_{2}$ are points on $C$. But (8.3) is obviously true for the finite set of the remaining $C_{m}$ 's.

9. Proof of Theorem V. Let

$$
s=\sigma_{m}(t)=\int_{0}^{t}\left|W_{m}^{\prime}(t)\right| d t, \quad 0 \leqq t \leqq T,
$$

denote the arc length along $C_{m}$. It follows from the Remark above that the functions $W_{m}^{(v)}(t)(\nu=1,2, \cdots, n)$ are equicontinuous and uniformly bounded for all $t$ and $m=1,2, \cdots$. Because of (8.2), the same is true for $1 / \sigma_{m}{ }^{\prime}(t)$.

Suppose first $n \geqq 2$. We have

$$
\kappa_{m}^{(1)}(s)=\kappa_{m}^{(1)}\left(\sigma_{m}(t)\right)=\frac{U_{m}^{\prime}(t) V_{m}^{\prime \prime}(t)-V_{m}^{\prime}(t) U_{m}^{\prime \prime}(t)}{\left(\sigma_{m}^{\prime}(t)\right)^{3}} .
$$

By differentiating $\kappa^{(1)}\left(\sigma_{m}(t)\right) \nu$ times with respect to $t$ we easily see that 
$\kappa_{m}^{(\nu)}(s)(\nu=2,3, \cdots, n-1)$ is a polynomial in $U_{m}^{(\mu)}(t), V_{m}^{(\mu)}(t)(\mu=1,2$, $\cdots, \nu+1)$ and in $1 / \sigma_{m}{ }^{\prime}(t)$. From this fact we infer first, with the help of (8.2), that the functions $\kappa_{m}^{(n-1)}(s)$, considered as functions of $s$, are equicontinuous and uniformly bounded for all $s$ and all $m=1,2, \ldots$. Furthermore, we infer from that fact and hypothesis (3), with the help of $\S 3,(1 \mathrm{a}),(3)$, and (2) and the inequality

$$
0<k_{0} \leqq \frac{\sigma_{m}(t)-\sigma_{m}\left(t^{\prime}\right)}{t-t^{\prime}} \leqq k_{1},
$$

that the integrals

$$
\int_{0}^{a}\left|\kappa_{m}^{(n-2)}(s+u)+\kappa_{m}^{(n-2)}(s-u)-2 \kappa_{m}^{(n-2)}(s)\right| \frac{d u}{u^{2}}
$$

exist and approach zero with $a$ uniformly for all $s$ and $m=1,2, \ldots$. Thus there exists a common convergence modulus for all these integrals.

If $n=1$, let $w_{m}(s)$ be the parametric representation of $C_{m}$, the arc length $s$ being the parameter. Let $\dot{w}_{m}(s)$ denote $d w_{m}(s) / d s$. Since

$$
\dot{w}_{m}(s)=\frac{W_{m}^{\prime}(t)}{\sigma_{m}{ }^{\prime}(t)}
$$

we see that the functions $w_{m}(s)$ are equicontinuous for all $s$ and all $m=1,2$, ... Furthermore, it follows from hypothesis (3) and (9.1) that

$$
\int_{0}^{a}\left|\dot{w}_{m}(s \pm u)-\dot{w}_{m}(s)\right| \frac{d u}{u}
$$

exists and approaches zero uniformly with $a$ for all $s$ and $m=1,2, \ldots$.

Since $W_{m}(t)$ converges uniformly toward $W(t)$ as $m \rightarrow \infty$, there is a number $D>0$, such that the diameter of every $C_{m}$ is $\leqq D$, and a number $\rho>0$, such that the circle with center $w_{0}$ and radius $\rho$ lies entirely in every $R_{m}(m=1$, $2, \cdots)$ and in $R$. Furthermore, (8.3) is true for every $C_{m}$, with $d$ independent of $m$. Therefore we may apply to every $C_{m}$ Theorem III (c) if $n>1$ and an analogous theorem about the first derivative $\dagger$ if $n=1$. According to these theorems the functions $f_{m}{ }^{(n)}(z)$ assume continuous boundary values over all of $|z|=1$, which are equicontinuous in $z$ and $m=1,2, \cdots$ and uniformly bounded.

Therefore we may apply to the functions $f_{m}{ }^{(n)}\left(e^{i \theta}\right), 0 \leqq \nu \leqq 2 \pi$, a theorem of Ascoli, $\ddagger$ according to which every subsequence of the $f_{m}^{(n)}\left(e^{i \theta}\right)$ contains a

$\dagger$ See WR, p. 440.

$\ddagger$ See, for example, O. D. Kellogg, Foundations of Potential Theory, Berlin, Springer, 1929, p. 265. 
subsequence $\left[f_{m_{k}}{ }^{(n)}\left(e^{i \theta}\right)\right]$ which converges uniformly in $0 \leqq \theta \leqq 2 \pi$, as $k \rightarrow \infty$. Hence also $f_{m_{k}}{ }^{(n)}(z)$ approaches uniformly a continuous function $F(z)$ in $|z| \leqq 1$. According to Weierstrass's convergence theorem, $F(z)$ is analytic in $|z|<1$.

Now it follows from the fact that $W_{m}(t)$ approaches $W(t)$ uniformly, that, in every fixed circle $|z| \leqq \rho<1, f_{m}(z)$ converges uniformly toward $f(z)$, and therefore also that $f_{m}(n)(z)$ approaches $f^{(n)}(z)$ there. Hence, in $|z|<1$, $F(z) \equiv f^{(n)}(z)$, so that $f^{(n)}(z)$ has continuous boundary values on $|z|=1$.

Since every subsequence of the $f_{m}^{(n)}(z)$ contains a uniformly convergent subsequence approaching always the same limit function $f^{(n)}(z)$ in $|z| \leqq 1$, it follows that the sequence $f_{m}^{(n)}(z)$ itself approaches $f^{(n)}(z)$ uniformly in $|z| \leqq 1$. Hence the theorem is true.

COLUMBia UNIVERSITY,

New YoRk, N. Y. 